\title{
Targeting BACH2-FOS signaling overcomes chemoresistance via stromal microenvironment alterations in pediatric acute lymphoblastic leukemia
}

Han Zhang ( $\boldsymbol{\sim}$ jennifer_z@imbcams.com.cn )

Chinese Academy of Medical Sciences and Peking Union Medical College https://orcid.org/0000-00022684-512X

\section{Ruidong Zhang}

Beijing Children's Hospital, Capital Medical University

\section{Xueling Zheng}

Beijing Children's Hospital, Capital Medical University

\section{Ming Sun}

Chinese Academy of Medical Sciences \& Peking Union Medical College Institute of Medical Biology Jia Fan

Beijing Children's Hospital, Capital Medical University

\section{Chunlian Fang}

Kunming Children's Hospital

\section{Xin Tian}

Kunming Children's Hospital

\section{Huyong Zheng}

Beijing Children's Hospital, Capital Medical University

\section{Research}

Keywords: $\mathrm{BACH}$, acute lymphoblastic leukemia, childhood, FOS, bone marrow microenvironment, chemoresistance

Posted Date: September 23rd, 2020

DOl: https://doi.org/10.21203/rs.3.rs-77370/v1

License: (9) This work is licensed under a Creative Commons Attribution 4.0 International License. Read Full License 


\section{Abstract \\ Background}

Acute lymphoblastic leukemia (ALL) is an aggressive hematological cancer that mainly affects children. Relapse and chemoresistance result in treatment failure, underlining the need for improved therapies. BTB and CNC homology 2 (BACH2) is a lymphoid-specific transcription repressor recognized as a tumor suppressor in lymphomas, but little is known about the function and regulatory network of BACH2 in pediatric ALL (p-ALL).

\section{Methods}

We analyzed the clinical relevance of $B A C H 2$ in nearly 450 published p-ALL microarray data. The mRNA and protein levels of BACH2 were validated in an independent cohort of p-ALL samples. The roles of $\mathrm{BACH} 2$ in leukemogenesis were examined using cell growth and proliferation assays, cell cycle and BrdU assays, cell apoptosis and cell adhesion assays as well as in vivo mouse models. Multiplexed flow cytometric assay was utilized to detect the bone marrow environmental alterations. Luciferase activity assay and CUT\&Tag sequencing were applied to determine the downstream target of BACH2. The therapeutic effects of chemicals were evaluated in precursor B (pre-B) ALL cells, primary p-ALL cells and in vivo pre-B ALL-driven leukemia xenografts.

\section{Results}

We found aberrant BACH2 expression at newly diagnosis not only facilitated risk stratification of p-ALL but also served as a sensitive predictor for early treatment response and clinical outcome. Silencing $\mathrm{BACH} 2$ in pre-B ALL cells increased cell proliferation and accelerated cell cycle progression. BACH2 blockade also promoted cell adhesion to bone marrow stromal cells and conferred chemo-resistant properties to leukemia cells by altering stromal microenvironment. Strikingly, we identified FOS, a transcriptional activator competing with $\mathrm{BACH} 2$, as a novel downstream target repressed by $\mathrm{BACH} 2$. Blocking FOS by chemical compounds enhanced the effect of cytarabine treatment in both primary p-ALL cells and pre-B ALL-driven leukemia xenografts and prolonged survival of tumor-bearing mice.

\section{Conclusions}

Our results highlight an interconnected network of BACH2-FOS, disruption of which could render current ALL chemotherapies more effective and offer a promising therapeutic strategy to overcome chemoresistance in $\mathrm{p}-\mathrm{ALL}$.

\section{Background}


BTB and CNC homology 2 (BACH2) is a lymphoid-specific transcription repressor encoded by the $B A C H 2$ gene. $\mathrm{BACH} 2$ is expressed abundantly in $\mathrm{B}$ cells and plays critical roles in the development and differentiation of lymphocytes $(1-3)$. In common lymphoid progenitors, $\mathrm{BACH} 2$ promotes B-cell development by repressing myeloid program (4). In pre- $\mathrm{B}$ cells, $\mathrm{BACH} 2$ is vital in negative selection at the pre-B cell receptor checkpoint (5). At later stages, BACH2 and B cell lymphoma 6 (BCL6) cooperatively regulate germinal center B-cell development, and thus enabling immunoglobulin class-switch recombination and the somatic hypermutation of immunoglobulin genes $(1,2,6)$.

In addition to B-cell development, large genome-wide association studies have identified numerous single-nucleotide polymorphisms in the human $B A C H 2$ locus that are linked to multiple autoimmune and allergic diseases (6). The mechanism underlying susceptibility to diverse immune-mediated diseases unveils additional roles of $\mathrm{BACH} 2$ in $\mathrm{CD}^{+}{ }^{+} \mathrm{T}$-cell differentiation (7) and in maintaining the naïve state of $\mathrm{T}$ cells by suppressing effector memory $T$ cell related genes (8). Given its essential roles in the development of $\mathrm{B}$ and $\mathrm{T}$ lymphocytes, $\mathrm{BACH} 2$ becomes more attractive to haemato-oncologists in terms of its function in hematological malignancies.

Over the past decade, $\mathrm{BACH} 2$ has been gradually recognized as a tumor suppressor in some hematological neoplasms. For example. elevated levels of $\mathrm{BACH} 2$ in patients with diffuse large B-cell lymphoma predict favorable outcomes (9). Enforced expression of $\mathrm{BACH} 2$ in Burkitt's lymphoma cell line RAJl remarkably inhibits cell proliferation and sensitizes cells to chemotherapy drugs (10). In mantle cell lymphoma, reduced $\mathrm{BACH} 2$ is associated with poor outcome in patients and chemoresistance in cell lines (11). BACH2 has also been reported as a safeguard against leukemogenesis in leukemia $(5,12)$. One major mechanism of $\mathrm{BACH} 2$ downregulation in blood cancers is the loss of the long arm of chromosome $6(6 q)$ or the genetic lesions of its upstream activator paired box $5(5,12,13)$. Despite its tumorsuppressor like roles in lymphomas, the function and downstream signaling of $\mathrm{BACH} 2$ in pediatric acute lymphoblastic leukemia (ALL) have so far remained elusive.

Pediatric ALL ( $p-A L L$ ) accounts for $~ 75 \%$ of pediatric leukemia, which is the commonest cancer and one of the primary causes of death in children. In the past two decades, the overall survival rate of p-ALL has exceeded $90 \%$ in some developed countries $(14,15)$. Even though, treatment failure or relapsed events still occur in $15 \sim 20 \%$ of children with ALL (16). Two major obstacles include incomplete understanding of leukemogenesis and lack of effective molecular targets in p-ALL (14).

In the present study, we found $\mathrm{BACH} 2$ levels can serve as a predictive factor for clinical outcome and chemoresistance in p-ALL patients on the basis of nearly 450 published p-ALL microarray data and tested it in an independent cohort of $\mathrm{p}-\mathrm{ALL}$ samples. Further studies of $\mathrm{BACH} 2$ in pre-B ALL cell line and primary cells revealed a tumor-suppressor role of BACH2. Notably, we for the first time identified FOS as a downstream target of $\mathrm{BACH} 2$ in pre-B ALL. The interaction between $\mathrm{BACH} 2$ and FOS further enhanced cell adhesion to bone marrow (BM) and promoted chemoresistance by altering microenvironmental conditions. Blocking FOS activity by two chemicals significantly improved the efficiency of cytarabine 
(Ara-C) treatment, both in vitro and in vivo. Collectively, our study lay the groundwork for the future study on the signaling network of $\mathrm{BACH} 2$ in the pathogenesis and chemoresistance of $\mathrm{p}-\mathrm{ALL}$.

\section{Materials And Methods Patient samples}

BM $(n=11)$ and peripheral blood $(n=1)$ aspirates were obtained from children with ALL at the time of newly diagnosis (ND) approved by the Institutional Review Boards, with informed consent obtained from their parents or guardians in accordance with the Declaration of Helsinki. Patient characteristics are described in Supplementary Table 1. Mononuclear cells were isolated by density gradient separation (Histopaque®-1077-PREMIUM, Sigma-Aldrich, St Louis, MO, USA) and cryopreserved for later use.

\section{Cell lines and plasmids}

Human pre-B ALL cell line Nalm- 6 was obtained from DSMZ (Braunschweig, Germany). Human bone marrow stromal cell (BMSC) HS-5 was obtained from BNCC (Beijing, China). Cell lines were authenticated using short tandem repeats at CinoAsia Institute (Shanghai, China). Nalm-6 cells/primary cells from pALL samples and HS-5 cells were maintained under $5 \% \mathrm{CO}_{2}$ at $37^{\circ} \mathrm{C}$ and cultured in complete RPMI 1640 medium and complete DMEM medium (Gibco, Carlsbad, CA, USA), respectively, supplemented with $10 \%$ FBS and $100 \mathrm{U} . \mathrm{I} . / \mathrm{ml}$ penicillin-streptomycin.

pcDNA3.1-BACH2 plasmid was constructed by sub-cloning BACH2 coding sequencing from human BACH2 plasmid (GE Dharmacon, clone ID: PLOHS_100066339, Pittsburgh, PA, USA) and inserting into pcDNA3.1(+) vector at BamHI and Xhol sites. The construction of pcDNA3.1-BACH2 plasmid was further verified by sequencing.

\section{Microarray data analysis}

Microarray data from 284 children with p-ALL at ND and 4 BM samples from 4 healthy donors (17), 35 ND-Relapse (RE) matched pairs and 43 RE samples of p-ALL (18), as well as 173 p-ALL samples at ND (19) were downloaded from the GEO database (http://www.ncbi.nlm.nih.gov/geo/; GSE28497, GSE3912, and GSE635, respectively). Microarray data from 20 leukemia cell lines (20) were downloaded from the Oncomine database (https://www.oncomine.org). All above datasets were based on the same microarray platform (Human Genome U133A Array). The expression values for BACH2 and $F O S$ in each dataset were used for the further analysis.

\section{Lentivirus generation and infection}

293T cells were transfected with either lentiviral shRNAs specific for human BACH2 (GE Dharmacon, clone ID: V3LHS_363286 and V3LHS_409004, Pittsburgh, PA, USA), or a non-silencing lentiviral shRNA control plasmid (GE Dharmacon), or the open reading frame (ORF) of human BACH2 (GE Dharmacon, clone ID: PLOHS_100066339). Lentiviruses were collected 48 hours post-transfection. Cells were then 
infected with lentiviruses using polybrene $(8 \mu \mathrm{g} / \mathrm{ml})$. Lentiviral-transduced cells were selected with puromycin $(2 \mu \mathrm{g} / \mathrm{ml})$ or blasticidin $(5 \mu \mathrm{g} / \mathrm{ml})$ (InvivoGen, San Diego, CA, USA) for 14 days.

\section{Quantitative real-time PCR (qRT-PCR)}

Procedures for qRT-PCR were performed using a One Step SYBR PrimeScript PLUS RT-PCR kit (Takara, Kusatsu, Japan) according to the manufacturer's protocol. The relative expression level of each gene was normalized to the GAPDH by the method of $2^{-\triangle \Delta C t}$. The involved primers are shown as follows: FOS. $5^{\prime}-$ AgAatCCGAAGgGAAAgGAA-3', 5'-CTTCTCCTTCAGCAGGTTGG-3'. Primers for the BACH2 gene (11) and the GPADH gene (21) was provided as described before.

\section{Immunoblotting assay and semi-quantitative analysis}

Harvested cells were lysed to perform immunoblotting assay as previously described (22). The following antibodies were used for immunoblots: anti-BACH2, anti-FOS and anti-GAPDH (Cell Signaling, Danvers, MA, USA). Immunoblotting was subjected to semi-quantitative analysis using an ImageJ software. The relative expression of target proteins was normalized to GAPDH.

\section{Drug preparation}

Cytarabine (Ara-C, Pfizer) was supplied by the Department of Hematology and Oncology, Kunming Children's Hospital. Ara-C and cyclophosphamide (CTX, Sigma-Aldrich, St Louis, MO, USA) were dissolved in a pyrogen-free sterile $0.9 \% \mathrm{NaCl}$ solution and stored at $-20^{\circ} \mathrm{C}$. Nordihydroguaiaretic acid (NDGA) and curcumin (Sigma-Aldrich, St Louis, MO, USA) were prepared in DMSO and stored at $-20^{\circ} \mathrm{C}$. For in vivo injection, the stocks were further diluted in PBS.

\section{Cell cycle analysis and intracellular BrdU incorporation assay}

Cell cycle and intracellular BrdU incorporation were performed using $\mathrm{PI} / \mathrm{RNase}$ Staining Buffer and an APC BrdU Flow kit (BD Biosciences, San Jose, CA), respectively, as previously described (11). Staining cells were analyzed on a NovoCyte flow cytometer (ACEA Biosciences, San Diego, CA, USA).

\section{Cell proliferation and cell survival assays}

PKH26 dye (Sigma-Aldrich, St Louis, MO, USA) and PE Annexin V Apoptosis Detection kit (BD Biosciences, San Jose, CA) were used to detect cell proliferation and survival, as previously described (11), on a NovoCyte flow cytometer.

\section{Cell viability assay and $\mathrm{IC}_{50}$}

Leukemic cells were treated with Ara-C for 48 hours, and cytotoxicity was assessed with fluorometric method using CellTiter-Blue® (Promega, Madison, WI, USA), as previously described (22). The Hill-slope logistic model was used to calculate $\mathrm{IC}_{50}$ values using a CompuSyn software (ComboSyn, $\mathrm{NJ}$, USA).

\section{Cell adhesion assay}


Cell adhesion was performed as previously described (22). PKH26 dye intensity was measured using FlexStation3 (Molecular Devices, San Jose, CA, USA). Representative pictures were taken using a Leika DMIL LED Fluorescence microscope (Leica, Germany). For neutralization experiments, human GM-CSF antibodies $(1 \mu \mathrm{g} / \mathrm{ml})$, human IL-8 antibodies $(1 \mu \mathrm{g} / \mathrm{ml})$, human $\mathrm{LL}-6$ antibodies $(0.5 \mu \mathrm{g} / \mathrm{ml})$ or lgG1 isotype control antibodies were used (R\&D systems, Minneapolis, MN, USA).

\section{Cytokines analysis}

Leukemic cells were plated onto the pre-established monolayer of HS-5 cells and allowed to coculture for 48 hours in $5 \% \mathrm{CO}_{2}$ at $37^{\circ} \mathrm{C}$ (complete RPMI1640: complete DMEM = 1:1). Coculture media were collected and measured by a multiplexed flow cytometric assay using a human cytokine kit (HCYTOMAG-60K-08) on a Luminex $\AA^{2}$ system (MAGPIX® with XPONENT). A total of 8 cytokines including G-CSF, GM-CSF, MIP$1 \mathrm{a}, \mathrm{IL}-1 \mathrm{a}, \mathrm{IL}-1 \beta, \mathrm{IL}-1 \mathrm{ra}, \mathrm{IL}-6$ and IL-8 were detected according to the instructions of the manufacturer (MILLIPLEX® Analyst 5.1). Samples were measured in duplicates with the coefficient of variation (CV) less than 20\%. Human GM-CSF (4A Biotech, Beijing, China), IL-6 and IL-8 (NeoBioscience, Shenzhen, China) were determined respectively by ELISA assays according to the manufacturer's protocols.

\section{Generation of truncated promoter constructs}

Three truncated human FOS promoters containing different numbers of putative MARE sites were amplified by PCR from genomic DNA. These PCR products were inserted into pGL3-basic vector (Promega, Madison, WI, USA) at Mlul and HindIII sites to make constructs of pGL3-MARE1, pGL3-MARE2, and pGL3-MARE3, respectively. These truncated promoter constructs were further verified by sequencing.

\section{Luciferase activity assay}

Luciferase activity was measured using a Dual-Luciferase Reporter Assay System kit (Promega, Madison, WI, USA) as described before (11). The data were normalized and presented as the ratio of firefly/Renilla luciferase activities.

\section{Cleavage Under Targets and Tagmentation (CUT\&Tag) assay}

Concanavalin A-coated magnetic beads bound to $60-100,000$ prepared living Nalm-6 cells by incubating at room temperature. The Hyperactive Tn5 transposon fused with Protein A/Protein G was precisely targeted to cut the DNA sequence near the target protein through the incubation of the primary human antibodies against $\mathrm{BACH} 2$ or control IgG, followed by incubation with a secondary antibody and Protein A/Protein G. DNA was sheared by the Tagmentation Buffer incubating at $37^{\circ} \mathrm{C}$. After DNA extraction, PCR amplification and PCR product purification, the libraries directly used for high-throughput sequencing and subsequent PCR validation was obtained.

\section{CUT\&Tag sequencing}

CUT\&Tag libraries were sequenced using an Illumina NovaSeq 6000 sequencer (Biomarker Technologies, Beijing, China). Raw Reads were filtered to remove adapters (Cutadapt software was used to remove the 
adapters and the reads less than $35 \mathrm{bp}$ in length) and low-quality reads (including Reads with $\mathrm{N}$ ratio greater than $10 \%$ and Reads with bases quality value $Q \leq 10$ accounting for more than $50 \%$ of the entire Read). High-quality Clean Reads provided in FASTQ format were obtained for subsequent information analysis. Integrative Genomics Viewer (IGV v2.8.3) software (23) was used to perform peak analysis using GRCh38 as a human genome reference.

\section{Tumor xenograft model}

BALB/C nude mice (5 weeks old) were purchased from Charles River Laboratories (Beijing, China), and were housed in the barrier conditions at Institute of Medical Biology (IMB). All animal procedures were approved by the IMB Animal Care Committee. All mice were pre-treated with an intraperitoneal (i.p.) injection of CTX at a dose of $100 \mathrm{mg} / \mathrm{kg}$ once daily for two consecutive days (24). Mice were then injected intravenously (i.v.) with manipulated Nalm- 6 cells via tail vein $\left(5 \times 10^{6}\right.$ cells/mouse, $n=3 /$ group $)$. Seven days post transplantation, xenograft mice were humanely sacrificed; bone marrow (BM) and spleen (SP) cells were collected to analyze leukemia engraftment by determining the percentage of positive human CD19 $\left(\mathrm{hCD}^{+} 9^{+}\right)$cells as previously described (11).

\section{In vivo Chemotherapy treatment}

Ten days following transplantation of $\mathrm{BACH} 2^{\mathrm{KD}}$ Nalm- 6 cells, mice were further split into four groups $(\mathrm{n}=$ 3/group). Different groups were i.p. injected with PBS (control), Ara-C alone, Ara-C + NDGA, Ara-C + curcumin, or Ara-C + both. Ara-C (100 mg/kg, once daily) (25), NDGA (100 mg/kg, twice daily) or curcumin (150 mg/kg, twice daily) (26) were administered for three consecutive days. Two days following the final dose of treatment, the mice were humanely sacrificed. BM and SP cells were collected and leukemic burden was evaluated by determining the percentage of $\mathrm{hCD} 19^{+}$cells as previously described (11). In another separate cohort, mice ( $n=5 /$ group) were treated to a humane endpoint with PBS, Ara-C, Ara-C + NDGA, Ara-C + curcumin, or Ara-C + both following the same protocol, and survival was assessed by Kaplan Meier analysis.

\section{Drug combination assay}

The synergic cytotoxic effects of NDGA/curcumin and Ara-C were determined by combination index (Cl) method as previously described (27). Cl plots were generated using a CompuSyn software. Briefly, synergy is present when the $\mathrm{Cl}$ is less than 1.0, additive effect is when $\mathrm{Cl}$ equals 1.0 , and antagonism is when $\mathrm{Cl}$ greater than 1.0 .

\section{Statistical analysis}

Data reported are described as experimental mean \pm standard error of mean (SEM) or standard deviation (SD). Statistical significance of differences between control and experimental groups was evaluated by the Student $t$ test, where ${ }^{\star} p<0.05,{ }^{*} p<0.01$ and ${ }^{* \star} p<0.001$ are considered statistically significant. All experiments and assays were repeated at least twice and performed in duplicate or triplicate. 


\section{Results}

\section{Expression feature of $\mathrm{BACH} 2$ is associated with risk stratification and early treatment responses}

To determine the clinical relevance of $\mathrm{BACH} 2$ in $\mathrm{p}-\mathrm{ALL}$, we firstly analyzed the expression values of $B A C H 2$ based on one published microarray data from 284 children with $A L L$ at newly diagnosis (ND). Compared with normal $\mathrm{BM} \mathrm{CD} 19^{+} \mathrm{CD} 10^{+}$cells from 4 healthy donors, leukemic cells from $\mathrm{p}-\mathrm{ALL}$ samples showed reduced $B A C H 2$ levels (Fig. 1A). Interestingly, we found lower $B A C H 2$ levels in patients with T-cell ALL (T-ALL) who have poorer outcomes than those with B-cell ALL (B-ALL) (28) (Fig. 1B). Among B-ALL samples, patients with unfavorable $B C R-A B L 1$ fusion gene contained remarkably lower $B A C H 2$ levels than patients without $B C R-A B L 1$ (Fig. 1C). In contrast, $B A C H 2$ expression in patients who have favorable

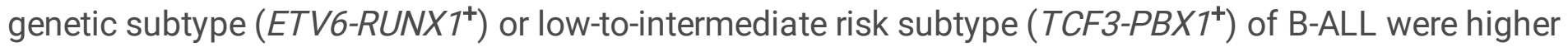
compared with patients who did not (Supplementary Fig. 1A and Fig. 1D-E), suggesting that differential expression of $B A C H 2$ could facilitate risk classification of $\mathrm{p}-\mathrm{ALL}$.

Given that minimal residual disease (MRD) tracking plays a crucial role in early outcome prediction for $\mathrm{p}$ $\mathrm{ALL}$, we next analyzed the correlation between $B A C H 2$ expression and MRD response. As shown in Fig. $1 F$, patients with lower $B A C H 2$ levels at ND were more inclined to occur positive MRD $\left(\mathrm{MRD}^{+}\right)$at day 19 (d19) from diagnosis (Fig. 1F), and this correlation becomes more significant at d46 (Fig. 1G).

Intriguingly, $\mathrm{MRD}^{+}$at d19 turned into $\mathrm{MRD}$ negative $\left(\mathrm{MRD}^{-}\right)$at $\mathrm{d} 46$ in patients with higher $B A C H 2$ levels in B-ALL compared with those with lower $B A C H 2$ levels, and the same is true in T-ALL (Supplementary Fig. 1B-C). Strikingly, $B A C H 2$ levels in different subtypes at ND also coincided with the MRD monitoring at d19 or d46. For example, the highest BACH2 levels were observed in patients with TCF3-PBX1 (Fig. 1E), while the smallest proportion of $\mathrm{MRD}^{+}$patients were found at either d19 or d46 (Supplementary Fig. 1D). These data suggest that aberrant expression of $B A C H 2$ at ND is very likely a predictor for early treatment response.

In addition to ND samples, further analysis from another microarray dataset revealed that early MRD response is also predictive for the degree of $B A C H 2$ expression at relapse $(\mathrm{RE})$ : the higher percentages of MRD at d36, the lower levels of $B A C H 2$ at RE $($ Fig. $1 \mathrm{H})$, and a much stronger inverse correlation between $\% \mathrm{MRD}$ and $B A C H 2$ expression was observed in T-ALL (Supplementary Fig. 1E), suggesting a reciprocal dependency of $B A C H 2$ and MRD on their respective role in outcome prediction.

\section{$\mathrm{BACH} 2$ is a sensitive predictor of clinical outcome}

To validate above microarray analysis, we examined the mRNA and protein levels of BACH2 in an independent cohort of $\mathrm{p}$-ALL samples at ND $(n=12)$. Indeed, $B A C H 2$ levels were significantly lower in $p$ ALL compared with patients with immune thrombocytopenic purpura (ITP), a non-tumorous hematologic disorder of megakaryocyte without disturbing lymphocytes (Fig. 2A). Of note, in addition to a patient with $T$-ALL, there is one patient with B/T-cell mixed-phenotype acute leukemia (B/T MPAL), a high-risk subtype of ALL with a uniformly poor outcome (29). BACH2 levels were much lower in T-ALL and B/T MPAL cases 
compared to B-ALL cases (Fig. 2B), and the lowest expression of BACH2 was observed in a B/T MPAL patient (Fig. 2C). Interestingly, amongst B-ALL cases, there is one special case (Pt \#12) that showed the lowest levels of $B A C H 2$ compared with the others (Fig. 2D). When reviewing the clinical information for this patient, we discovered that she had a very high tumor burden in peripheral blood at ND $(90 \%$ of blasts), and passed away soon after induction therapy (Supplementary Table 1). This, together with a close relationship between $B A C H 2$ and MRD as indicated above, further supported the possibility that $\mathrm{BACH} 2$ may serve as a sensitive predictor for risk classification and clinical outcome, although additional evidence from more clinical samples are needed.

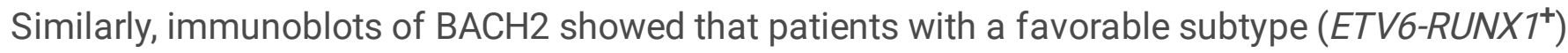
exhibited higher expression of $\mathrm{BACH} 2$ compared with patients with unfavorable subtypes $\left(B C R-A B L 1^{+}\right.$, B/T MPAL and T-ALL) (Fig. 2E), in agreement with both the microarray analysis and the mRNA findings.

\section{Silencing BACH2 increases leukemic cell proliferation and accelerates cell cycle progression}

To better delineate the biological roles of $\mathrm{BACH} 2$ in leukemic cells, we silenced $\mathrm{BACH} 2\left(\mathrm{BACH} 2^{\mathrm{KD}}\right)$ in a human pre-B ALL cell line using a lentiviral shRNA-mediated knockdown system. The knockdown efficiency of $\mathrm{BACH} 2$ in leukemic cells was evaluated by immunoblots which showed a better knockdown efficiency of $\mathrm{BACH} 2^{\mathrm{KD}}-2$ than $\mathrm{BACH} 2^{\mathrm{KD}}-1$ (Fig. $3 \mathrm{~A}$ ); $\mathrm{BACH} 2^{\mathrm{KD}}-2$ was selected to perform the subsequent experiments. Correspondingly, we generated stable $\mathrm{BACH} 2$-overexpressing $\left(\mathrm{BACH}{ }^{\mathrm{OE}}\right)$ cells (Fig. $\left.3 \mathrm{~A}\right)$.

Compared with control cells ( $\mathrm{BACH} 2^{\mathrm{Con}}$ ), silencing $\mathrm{BACH} 2$ significantly increased cell growth, whereas $\mathrm{BACH} 2{ }^{\mathrm{OE}}$ cells showed lower growth rate than the control (Fig. 3B), indicating a potential anti-tumor role of $\mathrm{BACH} 2$ in the pathogenesis of leukemia. To elucidate the mechanism involving enhanced cell growth in $\mathrm{BACH} 2^{\mathrm{KD}}$ cells, cell proliferation was analyzed by staining cells with $\mathrm{PKH} 26$ dye to track cell division. $\mathrm{PKH} 26$ fluorescent labelling was declined rapidly after $\mathrm{BACH} 2$ silencing while slowly in $\mathrm{BACH} 2{ }^{\mathrm{OE}}$ cells, indicating that downregulation of BACH2 promotes cell proliferation (Fig. 3C). Further analysis of cell cycle distribution showed approximately $15 \%$ more of $\mathrm{BACH} 2{ }^{\mathrm{KD}}$ cells in S-G2/M phases compared with control cells, while the diminished proliferation in $\mathrm{BACH} 2^{\mathrm{OE}}$ cells was associated with decreased S-G2/M population and increased apoptotic Sub-G1 population (Fig. 3D). Intracellular pulse staining for BrdU incorporation further confirmed higher amounts of $\mathrm{BACH} 2^{\mathrm{KD}}$ cells while lower amounts of $\mathrm{BACH} 2^{\mathrm{OE}}$ in $\mathrm{S}$ phase (Fig. 3E). These data indicated that silencing BACH2 leads to increased cell proliferation and accelerated cell cycle progression, thus contributing to a dominant growth.

To demonstrate in vivo relevance, we intravenously transplanted manipulated Nalm- 6 cells into mice (Supplementary Fig. 2). $\mathrm{BACH} 2^{\mathrm{KD}}$ xenografts developed larger spleens (SP) as compared to the $\mathrm{BACH} 2^{\mathrm{OE}}$ and control xenografts (Fig. 3F). Further analysis or these xenografts displayed increased human CD19+ $\left(\mathrm{hCD} 19^{+}\right)$cells in the SP and $\mathrm{BM}$ upon $\mathrm{BACH} 2$ silencing, indicating higher leukemia burden in $\mathrm{BACH} 2^{\mathrm{KD}}$ xenografts; by contrast, lower leukemia burden was observed in $\mathrm{BACH} 2^{\mathrm{OE}}$ xenografts (Fig. $3 \mathrm{G}$ ). 


\section{Decreased BACH2 expression confers chemo-resistant properties to $\mathrm{p}-\mathrm{ALL}$}

We next questioned the implication of reduced $B A C H 2$ levels in p-ALL treatment. Based on a published microarray data from $173 \mathrm{p}$-ALL cases at ND, patients with lower $B A C H 2$ levels predisposed to prednisolone resistant (Fig. 4A), and more obviously correlation was found in B-ALL group (Fig. 4B). In addition to clinical samples, leukemic cell lines with decreased $B A C H 2$ expression were also likely to occur cytarabine (Ara-C) resistance (Fig. 4C).

To confirm these findings, we tested whether $\mathrm{BACH} 2$ blockade contributes to chemoresistance in leukemic cells. Flow cytometry (FCM) analysis revealed a survival advantage of the $\mathrm{BACH}{ }^{\mathrm{KD}}$ cells compared to control cells, whereas higher proportion of apoptotic cells were found in the $\mathrm{BACH} 2^{\mathrm{OE}}$ cells (Fig. 4D), indicating that silencing BACH2 contributes to enhanced leukemic cell survival. After introducing Ara-C into leukemic cells, $\mathrm{BACH} 2$ deletion displayed lower drug sensitivity by preventing cell apoptosis, which was reversed by $\mathrm{BACH} 2$ overexpression (Fig. 4D). These data demonstrated that BACH2 downregulation confers Ara-C resistance properties to leukemic cells by likely increasing the threshold for drug-induced apoptosis.

\section{BACH2 silencing promotes cell adhesion and chemoresistance by altering stromal microenvironment}

Bone marrow stromal cells (BMSCs) are regarded as a safeguard to protect BM-resident leukemic cells from chemotherapy-induced apoptosis by producing multiple growth factors and cytokines, leading to stroma-mediated chemoresistance (30-33). Thus, BM microenvironmental remodeling has become a key parameter and prognostic factor in leukemia (34). Since $\mathrm{BACH} 2^{\mathrm{KD}}$ xenografts showed increased leukemia burden to the BM (Fig. $3 \mathrm{H}$ ), we then used a coculture model of leukemic cells and BMSCs to investigate the effect of $\mathrm{BACH} 2$ on cell adhesion and complex leukemia-stroma network, and how such effect modifies the cytotoxicity of anticancer drugs within the surrounding stroma.

Compared with control cells, silencing BACH2 in leukemic cells resulted in a significant increase in cell adhesion to the HS-5 BMSCs, while decreased cell adhesion was observed in $\mathrm{BACH}{ }^{\mathrm{OE}}$ cells (Fig. $5 \mathrm{~A}$ and Supplementary Fig. 3A). Further analysis using coculture media based on a multiplexed flow cytometric system revealed substantial changes in the secretion of many growth factors and cytokines that play pivotal roles in maintenance of normal BM microenvironment (35-37) (Fig. 5B). Coculturing HS-5 with $\mathrm{BACH} 2^{\mathrm{KD}}$ cells resulted in significant upregulation of GM-CSF, IL- 6 and IL-8 compared with control, whereas IL-6, IL-8 and MIP-1 a were decreased when coculturing BMSCs with $\mathrm{BACH} 2{ }^{\mathrm{OE}}$ cells (Supplementary Fig. 3B). These results were further validated by ELISA assays respectively for single cytokine from independent experiments (Supplementary Fig. 3C). As a result, the coculture media showed protective effects against Ara-C with much higher $\mathrm{IC}_{50}$ values, no matter in control or $\mathrm{BACH} 2^{\mathrm{KD}}$ cells 
(Fig. 5C), suggesting that BMSCs-secreted cytokines are very likely involved in Ara-C resistance of BMresident leukemic cells.

To extend our findings to primary cells, we performed experiments with BM cells from two p-ALL patients using a similar coculture setting. Primary cells or drug-resistant $\mathrm{BACH} 2^{\mathrm{KD}}$ cells did get great benefit from these secreted cytokines, because neutralization of IL-8 in coculture media led to decreased cell adhesion to BMSCs (Fig. 5D), whereas GM-CSF- or IL-6-neutralizing antibodies increased Ara-C-derived cytotoxicity (Fig. 5E). These results indicated that stromal microenvironmental alterations have many tumorpromoting effects that not only enhance cell adhesion, but also protect leukemic cells from chemotherapeutic-derived cytotoxicity in ALL.

\section{Proto-oncoprotein FOS is a novel downstream target repressed by BACH2 in pre-B leukemic cells}

$\mathrm{BACH} 2$ is a basic region leucine zipper (bZIP) protein that forms heterodimers with the small Maf proteins (38). The BACH2-Maf heterodimers repress transcription by binding to DNA sequences termed Maf recognition elements [MARE, 5'-TGCTGA(G/C)TCAGCA-3') (10,38). Interestingly, MARE includes a core TPA response element [TRE, $5^{\prime}-\mathrm{TGA}(\mathrm{G} / \mathrm{C}) \mathrm{TCA}-3^{\prime}$ ] that can be bound by activator protein 1 (AP-1), a dimeric transcriptional activator formed by JUN and FOS (6). Therefore, BACH2 regulates gene expression by competing with AP-1 complex (6). Despite well-established oncogenic functions of AP-1 signaling (3942), each component of AP-1 complex plays independent roles. JUN involves in tumorigenesis by regulating malignant transformation, apoptosis, angiogenesis and DNA methylation (43-47), whereas FOS, in addition to a similar role as JUN (48-50), plays an extra role in regulating bone cell differentiation and osteoimmunology (51). Mice lacking Fos develop severe osteopetrosis with deficiencies in bone remodeling (52) and exhibit altered B-cell differentiation due to an impaired BM microenvironment (53). In BCR-ABL1-induced leukemia, FOS was recently identified as one of critical mediators for leukemogenesis and imatinib resistance (26). Given a similar effect of $\mathrm{BACH} 2$ and FOS on BM microenvironmental regulation, we hypothesized that FOS might correlate with BACH2 in p-ALL.

To test this hypothesis, we started with the correlation analysis of $B A C H 2$ expression and FOS expression using published microarray data $(n=238)$, which showed a significant inverse correlation between them in B-ALL at ND (Fig. 6A). However, we did not find an inverse, but observed a positive correlation between $B A C H 2$ and FOS in T-ALL $(n=46)$ (Supplementary Fig. 4A), implying a totally different regulatory network of $\mathrm{BACH} 2$ in T cells.

Next, we detected the mRNA levels of $B A C H 2$ and FOS in clinical p-ALL samples, and the same inverse correlation was found in B-ALL at ND, except T-ALL and B/T MPAL cases (Supplementary Fig. 4B and Fig. 6B). Further immunoblots showed differential expression of FOS protein among different subtypes (Fig. 6C), exactly in contrary to the corresponding BACH2 levels in B-ALL group (Fig. 2E). In pre-B leukemic cells, FOS levels were also increased after $\mathrm{BACH} 2$ silencing while reduced in $\mathrm{BACH}{ }^{\mathrm{OE}}$ cells 
(Supplementary Fig. 4C). These results indicated that $\mathrm{BACH} 2$ is very likely a potential suppressing regulator of FOS in pre-B leukemic cells.

We next wondered whether the FOS gene is a transcriptional target repressed by BACH2 protein. Based on sequence alignment methods, we identified three potential MARE binding sites of the FOS gene within \pm $1000 \mathrm{bp}$, which are located at the proximal promoter (MARE1, -212/-202), the $5^{\prime}$ untranslated region (MARE2, +32/+43) and the proximal Exon 1 (MARE3, +181/+192), respectively (Fig. 6D). Truncated luciferase reporters containing different numbers of putative MAREs were constructed respectively. The luciferase activity of pGL3-MARE3 was significantly decreased in cells when co-transfected with BACH2 expression plasmids compared with the controls (Fig. 6D). Further sequencing using CUT\&Tag, a nextgeneration technique to investigate interactions between proteins and DNA instead of a chromatin immunoprecipitation (ChIP) assay, in pre-B Nalm-6 cells confirmed BACH2-FOS interaction (Fig. 6E). Indeed, two most enriched regions (fragments $b$ and $c$ ) of the FOS gene containing BACH2-binding sites were further validated by PCR amplification (Supplementary Fig. 5 and Fig. 6F). These findings support that BACH2 suppresses FOS transcription by binding to MARE sites on proximal regions of the FOS gene.

\section{Blocking FOS by small molecule inhibitors sensitizes leukemic cells to Ara-C in xenografts}

Given that the FOS gene is a downstream target repressed by $\mathrm{BACH} 2$, we then reasoned that FOS might be functionally involved in $\mathrm{BACH} 2$-induced $\mathrm{BM}$ microenvironmental alterations and chemoresistance. We firstly tested the effects of two chemical compounds targeting FOS, nordihydroguaiaretic acid (NDGA) and curcumin (26), on microenvironmental secretion of cytokines in a coculture setting of $\mathrm{BACH} 2^{\mathrm{KD}}$ cells and BMSCs. Both NDGA and curcumin were effective in suppressing the secretion of GM-CSF, IL-6 and IL8 in coculture media (Supplementary Fig. 6A). In particular, blocking FOS with NDGA or curcumin obviously sensitized leukemic cells to Ara-C treatment in coculture media, no matter in Ara-C-resistant $\mathrm{BACH} 2^{\mathrm{KD}}$ cells (Supplementary Fig. $6 \mathrm{~B}$ ) or in primary cells (Fig. 7A), suggesting that FOS is a very important mediator responsible for $\mathrm{BACH} 2$-induced microenvironmental changes and chemoresistance. The synergistic cytotoxic effects of Ara-C and NDGA/curcumin were further analyzed by combination index $(\mathrm{Cl})$ plots, which were all less than 1, indicating dramatic synergistic responses (Supplementary Fig. 7), in which, more synergistic efficacy of Ara-C and NDGA was observed than the combination of AraC with curcumin.

Next, we tested whether blocking FOS function is also effective in leukemia xenograft models. Ten days after intravenously transplantation with Ara-C-resistant $\mathrm{BACH} 2^{\mathrm{KD}}$ cells, mice were treated with Ara-C alone or in combination with NDGA (Ara-C $+\mathrm{N}$ ), curcumin (Ara-C $+\mathrm{C}$ ) or both (Ara-C + both) for 3 days and euthanized 2 days later (Fig. 7B). Mice treated with Ara- $\mathrm{C}+\mathrm{N}$ or Ara-C + both significantly reduced splenomegaly after one course of treatment (Fig. 7C). Combined treatment with Ara- $\mathrm{C}+\mathrm{N}$ or Ara-C + both also let to a more effective inhibition of leukemia burden in SP and BM compared to single-agent Ara-C group (Fig. 7D-E). In a separate cohort, mice treated with Ara-C $+\mathrm{N}$ or Ara-C + both showed improved 
survival compared to other groups (Fig. 7F), indicating that the combined treatment was effective to prolong survival of tumor-bearing animals.

\section{Discussion}

Despite incremental success in the treatment of p-ALL, much still remains to be achieved: not all patients receive optimal therapy, and cure rates remain modest or even poorer in high-risk groups such as $B C R$ $A B L 1^{+} \mathrm{B}-\mathrm{ALL}, \mathrm{T}-\mathrm{ALL}$ and MPAL cases. Tremendous advances have been witnessed in the understanding of the genetic basis of p-ALL by the application of next-generation sequencing technologies $(14,54)$, however, we are still far from fully deciphering the molecular pathogenesis of $\mathrm{p}-\mathrm{ALL}$ and the mechanisms underlying relapse and treatment failure.

In the current study, we found downregulation of $\mathrm{BACH} 2$ in children with unfavorable $B C R-A B L 1$ fusion gene compared with other subtypes in B-ALL. This finding is supported by a study demonstrating that $\mathrm{BACH} 2$ is a direct transcriptional target repressed by BCR-ABL1 oncoprotein via suppression of PAX5 expression in chronic myeloid leukemia cells (12). In contrast, we are surprised to observe much higher levels of $B A C H 2$ in $T C F 3-P B X 1^{+}$subtype at ND than a well-recognized favorable ETV6-RUNX $1^{+}$subtype (28), since ALL with $T C F 3-P B X 1$ was once associated with a poor prognosis. With contemporary MRDstratified therapy, $T C F 3-P B X 1^{+}$subtype is now classified as a low-to-intermediate risk genotype $(28,55)$. Children with TCF3-PBX1 treated with Berlin-Frankfurt-Münster (BFM) or Chinese Children's Leukemia Group (CCLG)-ALL protocols get even better event-free survival rates than those without TCF3-PBX1 (5658). The improved outcome of $T C F 3-P B X 1^{+}$subtype in $\mathrm{p}-A L L$ might be attributed to higher levels of $\mathrm{BACH} 2$ which facilitates efficient chemotherapies by sensitizing leukemic cells to agents. Indeed, we found ectopic expression of $\mathrm{BACH} 2$ in leukemic cells shows higher sensitivity to Ara-C, whereas downregulation of $\mathrm{BACH} 2$ confers Ara-C resistance properties to leukemic cells.

In addition, we discovered extremely lower levels of BACH2 in T-ALL and B/T MPAL cases. Unlike B-ALL, the genetic basis of T-ALL predisposition remains poorly understood, and no consensus genetic classification with prognostic or therapeutic implications has been reached for T-ALL, therefore the precision medicine approaches for T-ALL are lagging far behind in children (28). Facing a similar situation, B/T MPAL, a particularly rare and understudied subtype of ALL, defines a high-risk subgroup with an inferior outcome no matter what classification is used $(29,59)$. Although early MRD response is clinically useful in tailoring treatment, MRD detection is currently limited by their technical complexity. In this regard, the identification and molecular characterization of new oncogenes or tumor suppressors, such as BACH2, can provide new insights into the pathogenesis of T-ALL and B/T MPAL, yet offering a new opportunity for the development of therapeutic targets.

Despite our findings of $\mathrm{BACH} 2$ biological features in clinical samples and leukemic cells, it is still unclear whether $\mathrm{BACH} 2$ is an original cause or just a mediator of other causative factors and cellular perturbation in p-ALL. Here we found silencing BACH2 enhances adhesion of leukemic cells to BMSCs by upregulating GM-CSF, IL-6 and IL-8, which further led to chemoresistance within the surrounding stroma. These 
aberrant cytokines may not be the only abnormal factors in BM microenvironment for ALL. Rather, this finding provides a proof of concept that leukemic cells in BM have capacities for making interactions with BMSCs and activating aberrant signaling pathways, which may uniquely or collectively alter BM microenvironment, thus contributing to the survival and progression of ALL.

In fact, tumor suppressors such as p53 have proved difficult to target for cancer treatment, since development of reactivator drugs to recover the wild-type activity is much harder than designing drugs targeting cancer driver genes (60). The same is true for $\mathrm{BACH} 2$. Therefore, it requires new thinking or a different approach to target $\mathrm{BACH} 2$, such as targeting the downstream factors or co-factors of BACH2 instead. We found an inverse correlation between BACH2 and FOS in children with B-ALL. Of particular interesting to us, FOS, as well as being a transcriptional activator competing with $\mathrm{BACH}$, itself is also a downstream target repressed by $\mathrm{BACH} 2$. Our finding of $\mathrm{BACH} 2-\mathrm{FOS}$ signaling axis partially explains the microenvironmental alterations and leukemia chemoresistance in $\mathrm{BM}$, adding a new layer in the understanding of BACH2-mediated anti-cancer functions in p-ALL. Inspiringly, blocking FOS by NDGA or curcumin, either alone or in combination, remarkably synergized with Ara-C to battle against Ara-C-

resistant $\mathrm{BACH} 2{ }^{\mathrm{KD}}$ cells, especially in resistant coculture setting. Our experiments with $\mathrm{BACH}{ }^{\mathrm{KD}}$ leukemia xenografts treated with FOS inhibitors further provided strong evidence for this. These findings suggest a novel therapeutic strategy to efficiently overcome chemoresistance in p-ALL.

\section{Conclusions}

Collectively, aberrant BACH2 levels in p-ALL may serve as a novel indicator for risk stratification and early treatment responses. Our discovery of the inverse correlation between BACH2 and FOS provides the proof of $\mathrm{p}$-ALL progression and chemoresistance in surrounding BM stromal microenvironment. Future efforts to fully depict the regulatory network of $\mathrm{BACH} 2$ in $\mathrm{p}-\mathrm{ALL}$ will uncover more significant clues on the treatment of p-ALL and identify additional interactive partners of $B A C H 2$, if any, that are vital for pathogenesis of $\mathrm{p}$-ALL.

\section{Abbreviations}

ALL

acute lymphoblastic leukemia

AP-1

activator protein 1

Ara-C

cytarabine

BACH2

BTB and CNC homology 2

B-ALL

B-cell acute lymphoblastic leukemia

BCL6 
B cell lymphoma 6

BFM

Berlin-Frankfurt-Münster

BM

bone marrow

BMSC(s)

bone marrow stromal cell(s)

B/T MPAL

$B / T-c e l l$ mixed-phenotype acute leukemia

bZIP

basic region leucine zipper

CCLG

Chinese Children's Leukemia Group

ChIP

chromatin immunoprecipitation

$\mathrm{Cl}$

combination index

CUT\&Tag

cleavage under targets and tagmentation

FCM

flow cytometry

hCD19+

positive human CD19

IGV

integrative genomics viewer

IMB

Institute of Medical Biology

ITP

immune thrombocytopenic purpura

i.p.

intraperitoneal

i.v.

intravenous

MARE

Maf recognition elements

MRD

minimal residual disease

ND

newly diagnosis

NDGA 
nordihydroguaiaretic acid

p-ALL

pediatric acute lymphoblastic leukemia

pre-B

precursor $\mathrm{B}$

RE

relapse

SD

standard deviation

SEM

standard error of mean

SP

spleen

T-ALL

T-cell acute lymphoblastic leukemia

\section{Declarations}

\section{Ethics approval and consent to participate}

Informed consent was obtained from the parents or guardians of children with ALL in accordance with the Declaration of Helsinki. The experiments using patient samples were approved by the Institutional Review Boards from Beijing Children's Hospital and Kunming Children's Hospital, respectively. All in vivo animal procedures were approved by the IMB Animal Care Committee.

\section{Consent for publication}

All authors approved the submitted and final version.

\section{Availability of data and materials}

All data and materials in the current study are included in this paper and supplementary information.

\section{Competing interests}

The authors declare no conflict of interest.

\section{Funding}

This work was supported by grants from National Natural Science Foundation of China (NSFC) (No. 81900169), the Fundamental Research Funds for the Central Universities (No. 3332018130), and Natural 


\section{Author's contributions}

$\mathrm{H}$ Zhang performed the experiments and interpreted the data; RZ provided clinical samples and performed the clinical analyses; XZ, JF, CF and XT collected and processed clinical samples and assisted the clinical analyses; MS assisted the experiments. $\mathrm{H}$ Zheng assisted in project design. $\mathrm{H}$ Zhang designed the whole project, supervised the experiments and wrote the paper.

\section{Acknowledgments}

We would like to thank the families and each of the children with ALL who participated in this study.

\section{References}

1. Muto A, Tashiro S, Nakajima O, Hoshino H, Takahashi S, Sakoda E, et al. The transcriptional programme of antibody class switching involves the repressor Bach2. Nature. 2004;429(6991):56671.

2. Huang $\mathrm{C}$, Geng H, Boss I, Wang L, Melnick A. Cooperative transcriptional repression by BCL 6 and BACH2 in germinal center B-cell differentiation. Blood. 2014;123(7):1012-20.

3. Itoh-Nakadai A, Matsumoto M, Kato H, Sasaki J, Uehara Y, Sato Y, et al. A Bach2-Cebp Gene Regulatory Network for the Commitment of Multipotent Hematopoietic Progenitors. Cell Rep. 2017;18(10):2401-14.

4. Itoh-Nakadai A, Hikota R, Muto A, Kometani K, Watanabe-Matsui M, Sato Y, et al. The transcription repressors Bach2 and Bach1 promote B cell development by repressing the myeloid program. Nat Immunol. 2014;15(12):1171-80.

5. Swaminathan S, Huang C, Geng H, Chen Z, Harvey R, Kang H, et al. BACH2 mediates negative selection and p53-dependent tumor suppression at the pre-B cell receptor checkpoint. Nat Med. 2013;19(8):1014-22.

6. Igarashi K, Kurosaki T, Roychoudhuri R. BACH transcription factors in innate and adaptive immunity. Nat Rev Immunol. 2017;17(7):437-50.

7. Roychoudhuri R, Hirahara K, Mousavi K, Clever D, Klebanoff CA, Bonelli M, et al. BACH2 represses effector programs to stabilize T(reg)-mediated immune homeostasis. Nature. 2013;498(7455):50610.

8. Tsukumo S, Unno M, Muto A, Takeuchi A, Kometani K, Kurosaki T, et al. Bach2 maintains T cells in a naive state by suppressing effector memory-related genes. Proc Natl Acad Sci U S A. 2013;110(26):10735-40. 
9. Sakane-Ishikawa E, Nakatsuka S, Tomita Y, Fujita S, Nakamichi I, Takakuwa T, et al. Prognostic significance of BACH2 expression in diffuse large B-cell lymphoma: a study of the Osaka Lymphoma Study Group. J Clin Oncol. 2005;23(31):8012-7.

10. Kamio T, Toki T, Kanezaki R, Sasaki S, Tandai S, Terui K, et al. B-cell-specific transcription factor BACH2 modifies the cytotoxic effects of anticancer drugs. Blood. 2003;102(9):3317-22.

11. Zhang H, Chen Z, Miranda RN, Medeiros LJ, McCarty N. Bifurcated BACH2 control coordinates mantle cell lymphoma survival and dispersal during hypoxia. Blood. 2017;130(6):763-76.

12. Casolari DA, Makri M, Yoshida C, Muto A, Igarashi K, Melo JV. Transcriptional suppression of BACH2 by the Bcr-Abl oncoprotein is mediated by PAX5. Leukemia. 2013;27(2):409-15.

13. Sasaki S, Ito E, Toki T, Maekawa T, Kanezaki R, Umenai T, et al. Cloning and expression of human B cell-specific transcription factor BACH2 mapped to chromosome 6q15. Oncogene. 2000;19(33):3739-49.

14. Pui $\mathrm{CH}$, Nichols $\mathrm{KE}$, Yang JJ. Somatic and germline genomics in paediatric acute lymphoblastic leukaemia. Nat Rev Clin Oncol. 2019;16(4):227-40.

15. Pui $\mathrm{CH}$, Yang JJ, Hunger SP, Pieters R, Schrappe M, Biondi A, et al. Childhood Acute Lymphoblastic Leukemia: Progress Through Collaboration. J Clin Oncol. 2015;33(27):2938-48.

16. Hunger SP, Mullighan CG. Acute Lymphoblastic Leukemia in Children. N Engl J Med. 2015;373(16):1541-52.

17. Coustan-Smith E, Song G, Clark C, Key L, Liu P, Mehrpooya M, et al. New markers for minimal residual disease detection in acute lymphoblastic leukemia. Blood. 2011;117(23):6267-76.

18. Bhojwani D, Kang H, Moskowitz NP, Min DJ, Lee H, Potter JW, et al. Biologic pathways associated with relapse in childhood acute lymphoblastic leukemia: a Children's Oncology Group study. Blood. 2006;108(2):711-7.

19. Holleman A, Cheok MH, den Boer ML, Yang W, Veerman AJ, Kazemier KM, et al. Gene-expression patterns in drug-resistant acute lymphoblastic leukemia cells and response to treatment. $\mathrm{N}$ Engl J Med. 2004;351(6):533-42.

20. Garnett MJ, Edelman EJ, Heidorn SJ, Greenman CD, Dastur A, Lau KW, et al. Systematic identification of genomic markers of drug sensitivity in cancer cells. Nature. 2012;483(7391):570-5.

21. Zhang H, Chen Z, Miranda RN, Medeiros LJ, McCarty N. TG2 and NF-kappaB Signaling Coordinates the Survival of Mantle Cell Lymphoma Cells via IL6-Mediated Autophagy. Cancer Res. 2016;76(21):6410-23.

22. Zhang H, Chen Z, Neelapu SS, Romaguera J, McCarty N. Hedgehog inhibitors selectively target cell migration and adhesion of mantle cell lymphoma in bone marrow microenvironment. Oncotarget. 2016;7(12):14350-65.

23. Robinson JT, Thorvaldsdottir H, Winckler W, Guttman M, Lander ES, Getz G, et al. Integrative genomics viewer. Nat Biotechnol. 2011;29(1):24-6. 
24. Zhang J, Lu WY, Zhang JM, Lu RQ, Wu LX, Qin YZ, et al. S100A16 suppresses the growth and survival of leukaemia cells and correlates with relapse and relapse free survival in adults with Philadelphia chromosome-negative B-cell acute lymphoblastic leukaemia. $\mathrm{Br} \mathrm{J}$ Haematol. 2019;185(5):836-51.

25. Burt R, Dey A, Aref S, Aguiar M, Akarca A, Bailey K, et al. Activated stromal cells transfer mitochondria to rescue acute lymphoblastic leukemia cells from oxidative stress. Blood. 2019;134(17):1415-29.

26. Kesarwani M, Kincaid Z, Gomaa A, Huber E, Rohrabaugh S, Siddiqui Z, et al. Targeting c-FOS and DUSP1 abrogates intrinsic resistance to tyrosine-kinase inhibitor therapy in BCR-ABL-induced leukemia. Nat Med. 2017;23(4):472-82.

27. Jung HJ, Chen Z, McCarty N. Synergistic anticancer effects of arsenic trioxide with bortezomib in mantle cell lymphoma. Am J Hematol. 2012;87(12):1057-64.

28. Teachey DT, Pui CH. Comparative features and outcomes between paediatric T-cell and B-cell acute lymphoblastic leukaemia. Lancet Oncol. 2019;20(3):e142-54.

29. Wolach O, Stone RM. How I treat mixed-phenotype acute leukemia. Blood. 2015;125(16):2477-85.

30. Jacamo R, Chen Y, Wang Z, Ma W, Zhang M, Spaeth EL, et al. Reciprocal leukemia-stroma VCAM1/VLA-4-dependent activation of NF-kappaB mediates chemoresistance. Blood. 2014;123(17):2691702.

31. Bendall LJ, Kortlepel K, Gottlieb DJ. Human acute myeloid leukemia cells bind to bone marrow stroma via a combination of beta-1 and beta-2 integrin mechanisms. Blood. 1993;82(10):3125-32.

32. Matsunaga T, Takemoto N, Sato T, Takimoto R, Tanaka I, Fujimi A, et al. Interaction between leukemic-cell VLA-4 and stromal fibronectin is a decisive factor for minimal residual disease of acute myelogenous leukemia. Nat Med. 2003;9(9):1158-65.

33. Zeng Z, Samudio IJ, Munsell M, An J, Huang Z, Estey E, et al. Inhibition of CXCR4 with the novel RCP168 peptide overcomes stroma-mediated chemoresistance in chronic and acute leukemias. Mol Cancer Ther. 2006;5(12):3113-21.

34. Kim JA, Shim JS, Lee GY, Yim HW, Kim TM, Kim M, et al. Microenvironmental remodeling as a parameter and prognostic factor of heterogeneous leukemogenesis in acute myelogenous leukemia. Cancer Res. 2015;75(11):2222-31.

35. Roecklein BA, Torok-Storb B. Functionally distinct human marrow stromal cell lines immortalized by transduction with the human papilloma virus E6/E7 genes. Blood. 1995;85(4):997-1005.

36. Yin T, Li L. The stem cell niches in bone. J Clin Invest. 2006;116(5):1195-201.

37. Colmone A, Amorim M, Pontier AL, Wang S, Jablonski E, Sipkins DA. Leukemic cells create bone marrow niches that disrupt the behavior of normal hematopoietic progenitor cells. Science. 2008;322(5909):1861-5.

38. Oyake T, Itoh K, Motohashi H, Hayashi N, Hoshino H, Nishizawa M, et al. Bach proteins belong to a novel family of BTB-basic leucine zipper transcription factors that interact with MafK and regulate transcription through the NF-E2 site. Mol Cell Biol. 1996;16(11):6083-95. 
39. Young MR, Li JJ, Rincon M, Flavell RA, Sathyanarayana BK, Hunziker R, et al. Transgenic mice demonstrate AP-1 (activator protein-1) transactivation is required for tumor promotion. Proc Natl Acad Sci U S A. 1999;96(17):9827-32.

40. Jochum W, Passegue E, Wagner EF. AP-1 in mouse development and tumorigenesis. Oncogene. 2001;20(19):2401-12.

41. Liu Y, Ludes-Meyers J, Zhang Y, Munoz-Medellin D, Kim HT, Lu C, et al. Inhibition of AP-1 transcription factor causes blockade of multiple signal transduction pathways and inhibits breast cancer growth. Oncogene. 2002;21(50):7680-9.

42. Motiwala T, Zanesi N, Datta J, Roy S, Kutay H, Checovich AM, et al. AP-1 elements and TCL1 protein regulate expression of the gene encoding protein tyrosine phosphatase PTPROt in leukemia. Blood. 2011;118(23):6132-40.

43. Bakiri L, Lallemand D, Bossy-Wetzel E, Yaniv M. Cell cycle-dependent variations in c-Jun and JunB phosphorylation: a role in the control of cyclin D1 expression. EMBO J. 2000;19(9):2056-68.

44. Eferl R, Ricci R, Kenner L, Zenz R, David JP, Rath M, et al. Liver tumor development. c-Jun antagonizes the proapoptotic activity of p53. Cell. 2003;112(2):181-92.

45. Vleugel MM, Greijer AE, Bos R, van der Wall E, van Diest PJ. c-Jun activation is associated with proliferation and angiogenesis in invasive breast cancer. Hum Pathol. 2006;37(6):668-74.

46. Mariani O, Brennetot C, Coindre JM, Gruel N, Ganem C, Delattre O, et al. JUN oncogene amplification and overexpression block adipocytic differentiation in highly aggressive sarcomas. Cancer Cell. 2007;11(4):361-74.

47. Kollmann K, Heller G, Ott RG, Scheicher R, Zebedin-Brandl E, Schneckenleithner C, et al. c-JUN promotes BCR-ABL-induced lymphoid leukemia by inhibiting methylation of the 5 ' region of Cdk6. Blood. 2011;117(15):4065-75.

48. Wang ZQ, Grigoriadis AE, Mohle-Steinlein U, Wagner EF. A novel target cell for c-fos-induced oncogenesis: development of chondrogenic tumours in embryonic stem cell chimeras. EMBO J. 1991;10(9):2437-50.

49. Grigoriadis AE, Schellander K, Wang ZQ, Wagner EF. Osteoblasts are target cells for transformation in c-fos transgenic mice. J Cell Biol. 1993;122(3):685-701.

50. Milde-Langosch $\mathrm{K}$. The Fos family of transcription factors and their role in tumourigenesis. Eur $\mathrm{J}$ Cancer. 2005;41(16):2449-61.

51. Wagner EF, Eferl R. Fos/AP-1 proteins in bone and the immune system. Immunol Rev. 2005;208:12640.

52. Wang ZQ, Ovitt C, Grigoriadis AE, Mohle-Steinlein U, Ruther U, Wagner EF. Bone and haematopoietic defects in mice lacking c-fos. Nature. 1992;360(6406):741-5.

53. Okada S, Wang ZQ, Grigoriadis AE, Wagner EF, von Ruden T. Mice lacking c-fos have normal hematopoietic stem cells but exhibit altered B-cell differentiation due to an impaired bone marrow environment. Mol Cell Biol. 1994;14(1):382-90. 
54. Roberts KG, Mullighan CG. Genomics in acute lymphoblastic leukaemia: insights and treatment implications. Nat Rev Clin Oncol. 2015;12(6):344-57.

55. Iacobucci I, Mullighan CG. Genetic Basis of Acute Lymphoblastic Leukemia. J Clin Oncol. 2017;35(9):975-83.

56. Kager L, Lion T, Attarbaschi A, Koenig M, StrehI S, Haas OA, et al. Incidence and outcome of TCF3PBX1-positive acute lymphoblastic leukemia in Austrian children. Haematologica. 2007;92(11):1561-4.

57. Felice MS, Gallego MS, Alonso CN, Alfaro EM, Guitter MR, Bernasconi AR, et al. Prognostic impact of $\mathrm{t}(1 ; 19)$ / TCF3-PBX1 in childhood acute lymphoblastic leukemia in the context of Berlin-FrankfurtMunster-based protocols. Leuk Lymphoma. 2011;52(7):1215-21.

58. Hu Y, He H, Lu J, Wang Y, Xiao P, Li J, et al. E2A-PBX1 exhibited a promising prognosis in pediatric acute lymphoblastic leukemia treated with the CCLG-ALL2008 protocol. Onco Targets Ther. 2016;9:7219-25.

59. Mi X, Griffin G, Lee W, Patel S, Ohgami R, Ok CY, et al. Genomic and clinical characterization of B/T mixed phenotype acute leukemia reveals recurrent features and T-ALL like mutations. Am J Hematol. 2018;93(11):1358-67.

60. Duffy MJ, Synnott NC, O'Grady S, Crown J. Targeting p53 for the treatment of cancer. Semin Cancer Biol. 2020.

\section{Figures}


A

- Normal BM $(n=4)$

- Pediatric ALL at ND ( $n=284)$

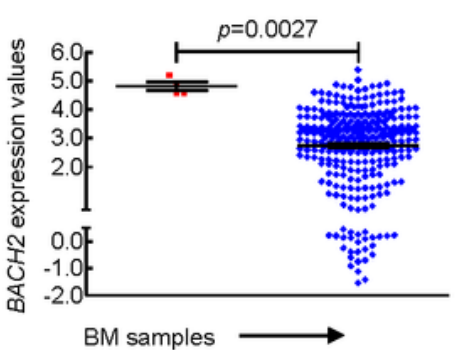

D

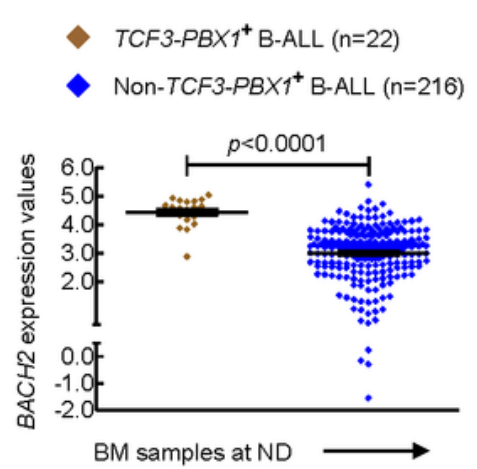

G

MRD ${ }^{+} B-A L L$ at $d 46(n=47)$

- MRD- B-ALL at d46 $(n=139)$

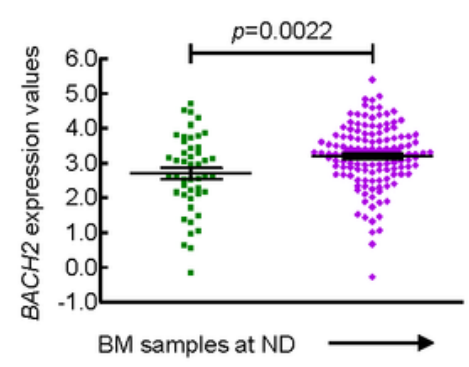

C

$\Delta B C R-A B L 1^{+}$B-ALL $(n=16)$

- Non-BCR-ABL1 $1^{+}$B-ALL $(n=222)$
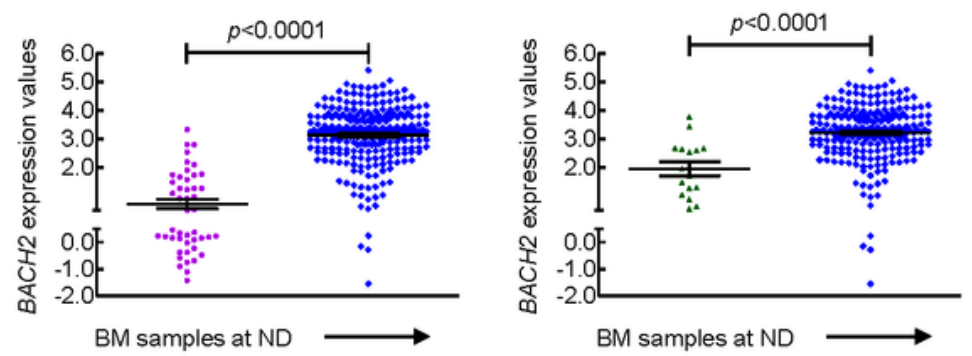

$\mathbf{F}$

- MRD ${ }^{+}$B-ALL at d19 $(n=97)$

- MRD ${ }^{-}$B-ALL at d19 $(n=73)$
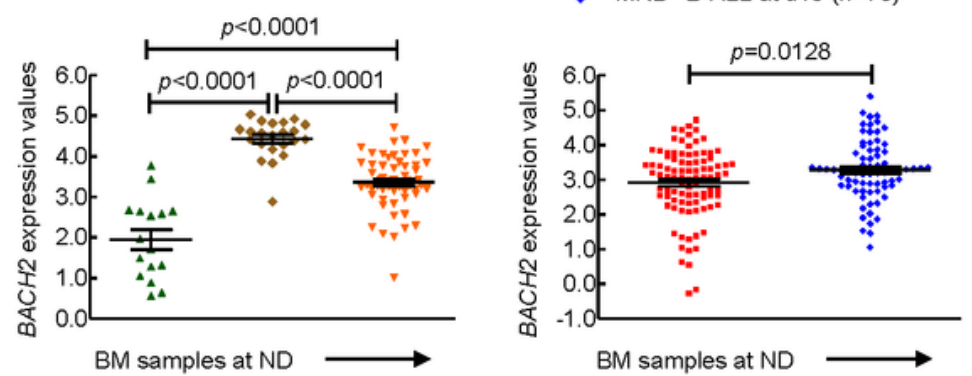

H

v MRD $<0.01 \%$ at $d 36(n=19)$

- $0.01 \%<M R D<1 \%$ at $d 36(n=12)$

- $1 \%<M R D<10 \%$ at $\mathrm{d} 36(\mathrm{n}=7)$

- MRD $>10 \%$ at $d 36 \quad(n=2)$

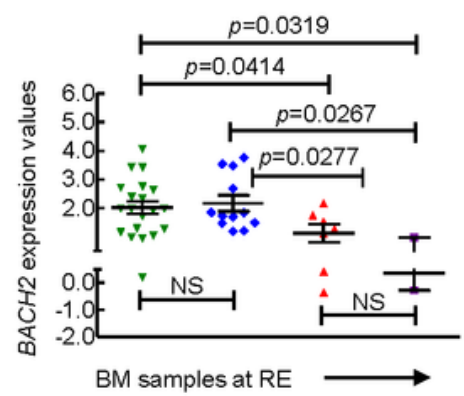

Figure 1

Expression feature of $\mathrm{BACH} 2$ levels in p-ALL from microarray data. (A) BACH2 mRNA levels were downregulated in BM cells from $p-A L L$ at ND $(n=284)$ compared with CD19+CD10+BM cells from healthy donors $(n=4)$. (B) BM cells from T-ALL $(n=46)$ contained lower levels of BACH2 than BM cells from B-ALL at ND ( $n=238)$. (C) BM cells from B-ALL with BCR-ABL1 fusion gene at ND $(n=16)$ contained lower levels of BACH2 compared with those from B-ALL without BCR-ABL1 fusion gene $(n=222)$. (D) BM cells from 
TCF3-PBX1 + B-ALL at ND ( $n=22)$ contained higher BACH2 levels than those from B-ALL without TCF3PBX1 fusion gene $(n=216)$. (E) Comparison of BACH2 levels in BM among different subtypes. B-ALL patients with lower $B A C H 2$ levels in $B M$ got MRD+ at d19 from diagnosis $(F)$, and remained MRD+ at day $46(G)$. (H) Inverse correlation between \%MRD and BACH2 levels in BM from RE samples at d36 (M1 patients). Above data are shown as the mean \pm SEM. ND, newly diagnosis; RE, relapse.

Figure 2

Zhang et al.

A

- ITP $(n=2)$

- Pediatric ALL $(n=12)$

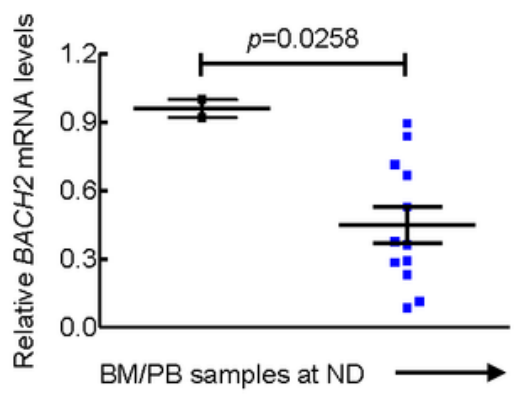

B

- T-ALL and B/T MPAL $(n=2)$

B-ALL $(n=10)$

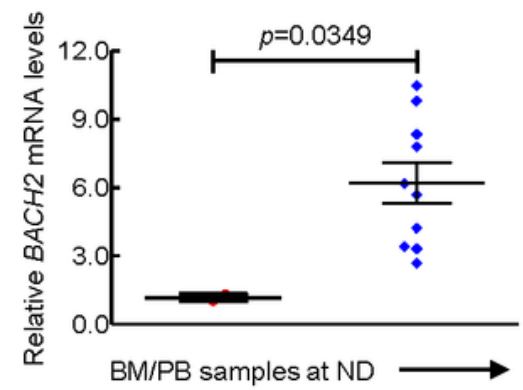

C

口 B/T MPAL

口 T-ALL

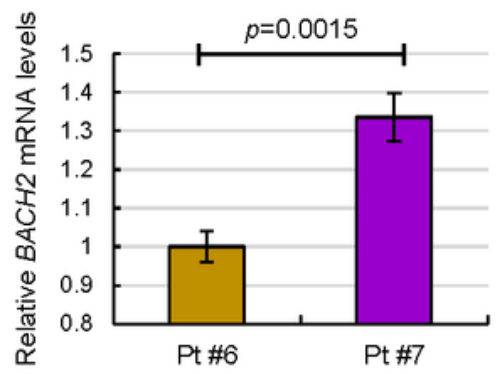

D

B-ALL $(n=10)$

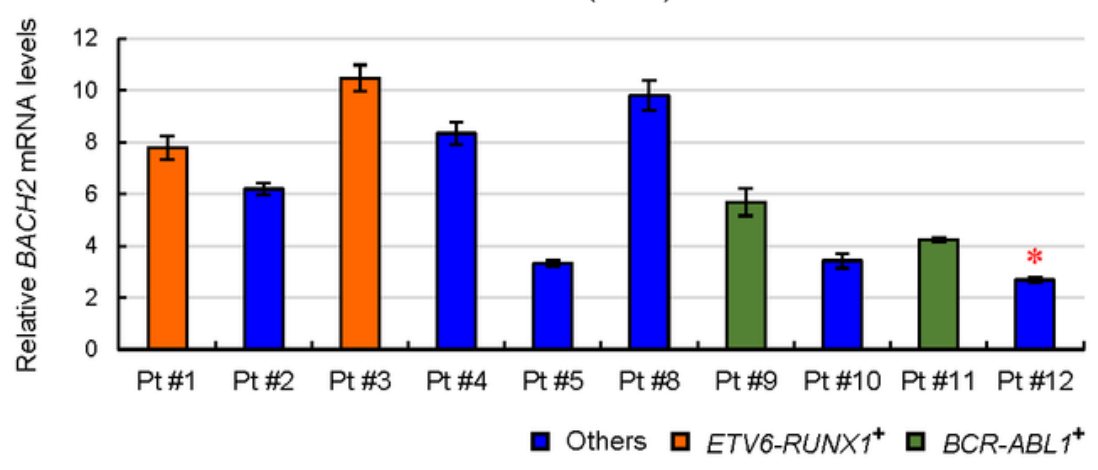

E

Pediatric ALL BM samples

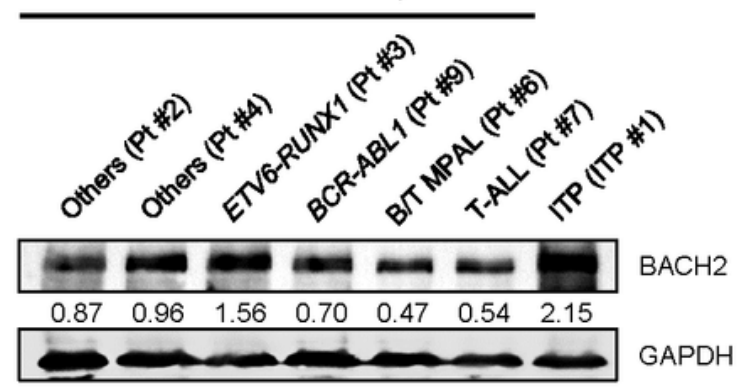

Figure 2 
Decreased BACH2 levels are associated with clinical outcome in p-ALL. (A) BACH2 mRNA levels were lower in $p$-ALL samples at ND $(n=12)$ compared with samples from patients with ITP $(n=2)$. Each condition was run in triplicate with the values normalized to GAPDH. The results are shown as the mean \pm SEM. (B) Leukemic cells in T-ALL and B/T MPAL $(n=2)$ contained lower levels of BACH2 than those from B-ALL $(n=10)$. The results are shown as the mean \pm SEM. (C) BACH2 levels in T-ALL and B/T MPAL. (D) BACH2 levels in B-ALL. The red asterisk indicates the patient \#12 (Pt \#12) with the lowest levels of $\mathrm{BACH}$ 2. (E) Immunoblots of BACH2 in different subtypes of p-ALL with one ITP sample as a negative control. GAPDH was used as a loading control. 
Figure 3

A

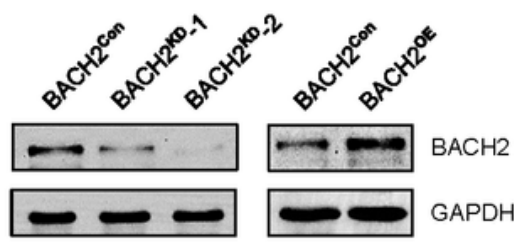

B

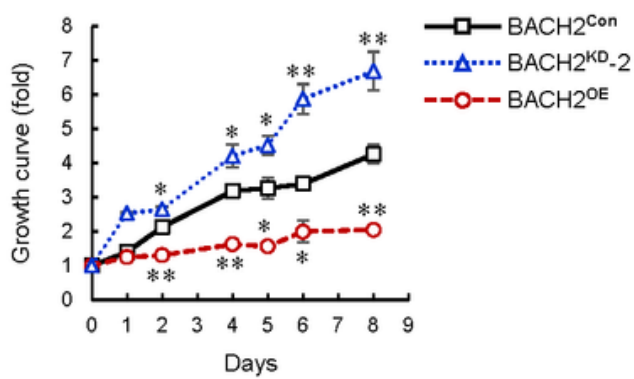

D
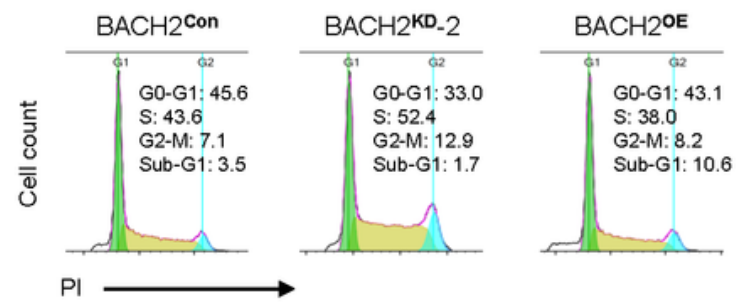

E

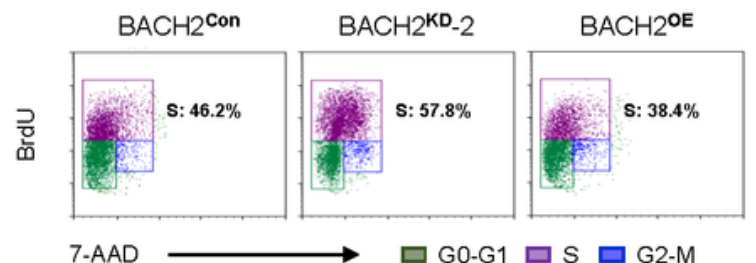

F

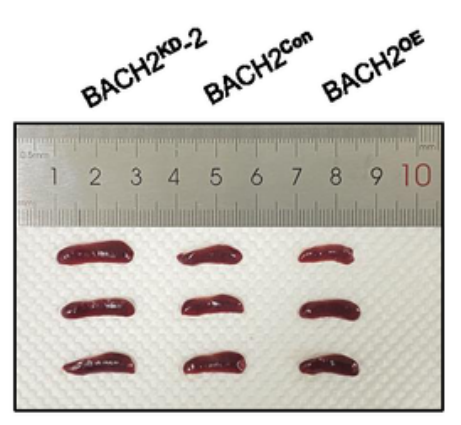

C
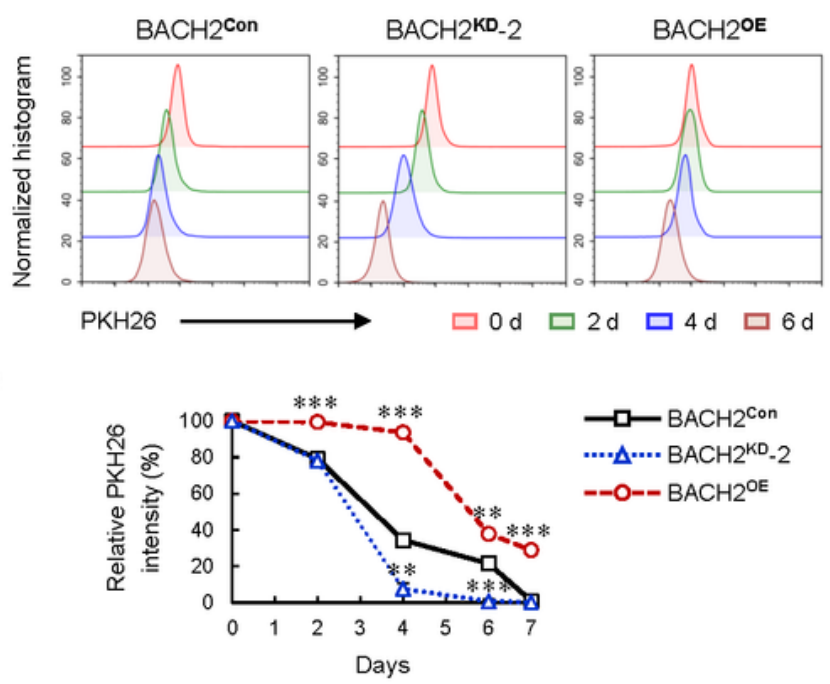

- $-\mathrm{BACH} 2^{\mathrm{Con}}$

$\cdots \cdot \cdot \cdots \mathrm{BACH}^{\mathrm{KD}}-2$

--O-- BACH2 ${ }^{\mathrm{OE}}$

$\square$ Go-G1 $\square$ S $\square$ G2-M $\square$ Sub-G1
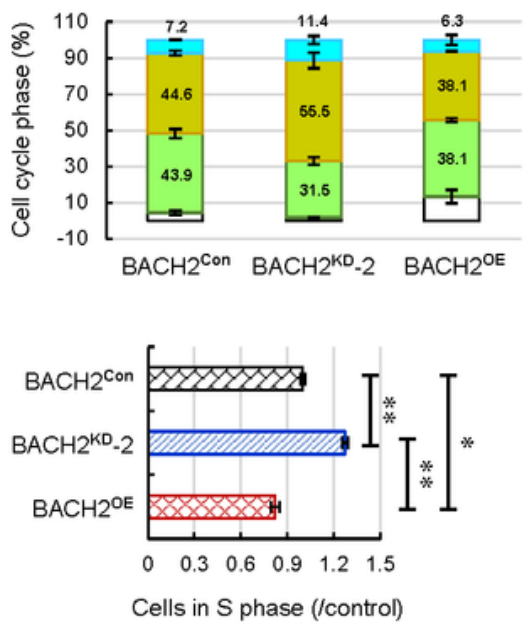

G

- $\mathrm{BACH}_{2}{ }^{\mathrm{Con}}(\mathrm{n}=3)$

- $B A C H 2^{\mathrm{KD}}-2(n=3)$

- $\mathrm{BACH} 2^{\mathrm{OE}}(\mathrm{n}=3)$
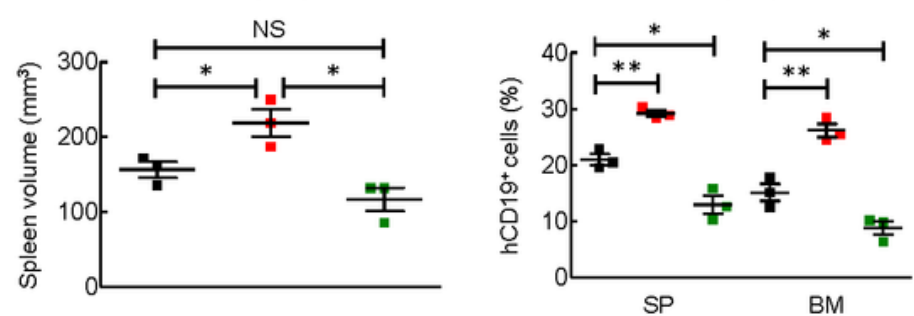

\section{Figure 3}

Silencing $\mathrm{BACH} 2$ increases cell proliferation and accelerates cell cycle progression. (A) The knockdown and overexpressed efficiency of $\mathrm{BACH} 2$ (BACH2KD and $\mathrm{BACH} 2 \mathrm{OE}$ ) in pre-B ALL cells were validated using immunoblots respectively with a non-silencing shRNA plasmid (BACH2Con) as a negative control. GAPDH was used as a loading control. (B) Viable cells were counted in manipulated pre-B ALL cells. (C) Manipulated ALL cells were stained with PKH26 fluorescent dye which was analyzed using FCM. 
Representative FCM analyses at 0, 2, 4 and 6 are shown (upper). The relative PKH26 intensity in each group is shown (lower). (D) Representative cell-cycle distribution of BACH2Con, BACH2KD-2 and BACH2OE cells (left), with the \% population of cells in each cell cycle phase is shown (right). (E) Representative intracellular pulse staining of BrdU in manipulated ALL cells (left). The population of $\mathrm{BACH} 2 \mathrm{KD}-2$ or BACH2OE cells in S phase was normalized to the control cells (right). Above data are shown as the mean \pm SD from at least 2 independent experiments. ${ }^{*} p<0.05 ;{ }^{* \star} p<0.01 ;{ }^{* \star *} p<0.001$ (vs control group; Student $t$ test). (F) BACH2KD-2, BACH2OE or BACH2Con cells were intravenously injected into mice $(n=3)$. Xenografts were humanely sacrificed 7 days post transplantation, and spleens (SP) were isolated and photographed against a ruler (left). The size of spleens in each group were measured (right). The results are shown as the mean \pm SEM. NS, not significant; ${ }^{*} \mathrm{p}<0.05$ (Student $t$ test). (G) Human CD19+ (hCD19+) cells were isolated from SP and BM using anti-hCD19-MicroBeads, and the number of hCD19+ cells was counted. The percentage of hCD19+ cells in each organ was calculated, and the data are presented as the mean \pm SEM. ${ }^{*} p<0.05 ;{ }^{*} p<0.01$ (vs control xenografts; Student $t$ test).

\section{Figure 4}

Zhang et al.

A

$\triangle \quad$ Prednisolone resistant $(n=27)$

$\triangle$ Prednisolone sensitive $(n=66)$

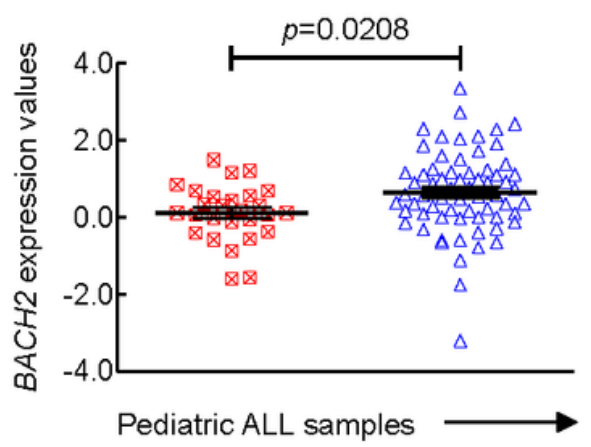

D

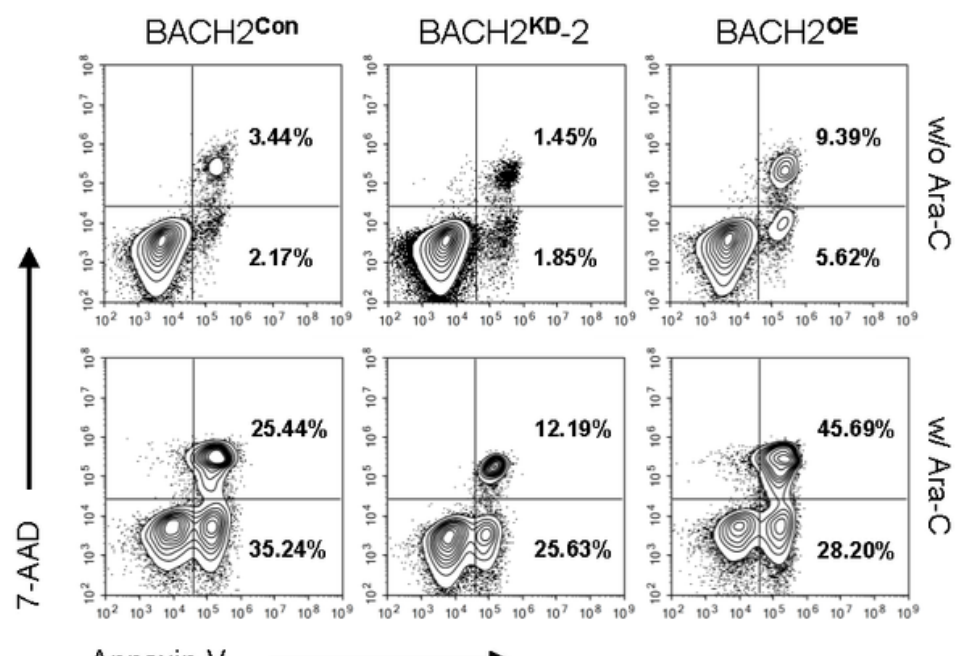

C

$\otimes$ Prednisolone resistant $(n=20)$

$\triangle$ Prednisolone sensitive $(n=55)$
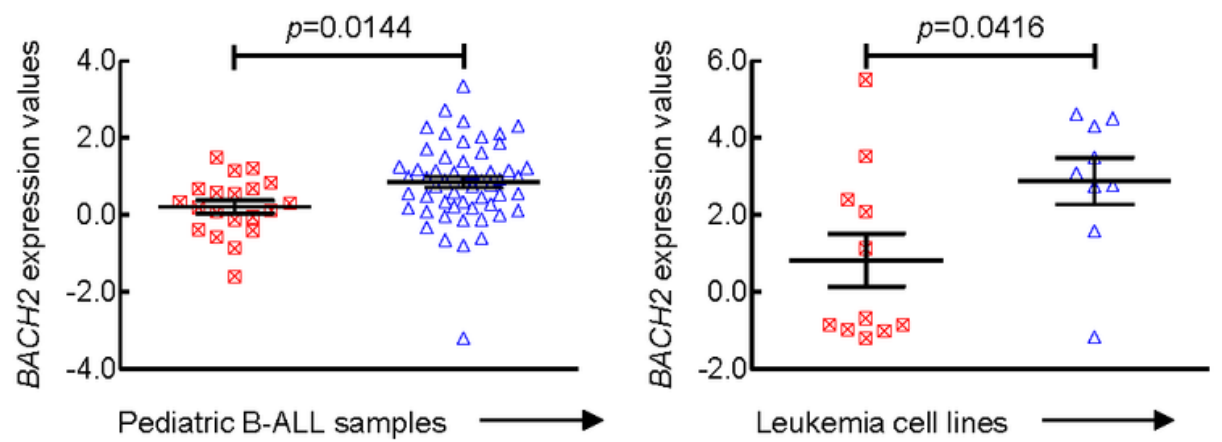

$\otimes \quad$ Ara-C resistant $(n=11)$

$\triangle$ Ara-C sensitive $(n=9)$

Leukemia cell lines

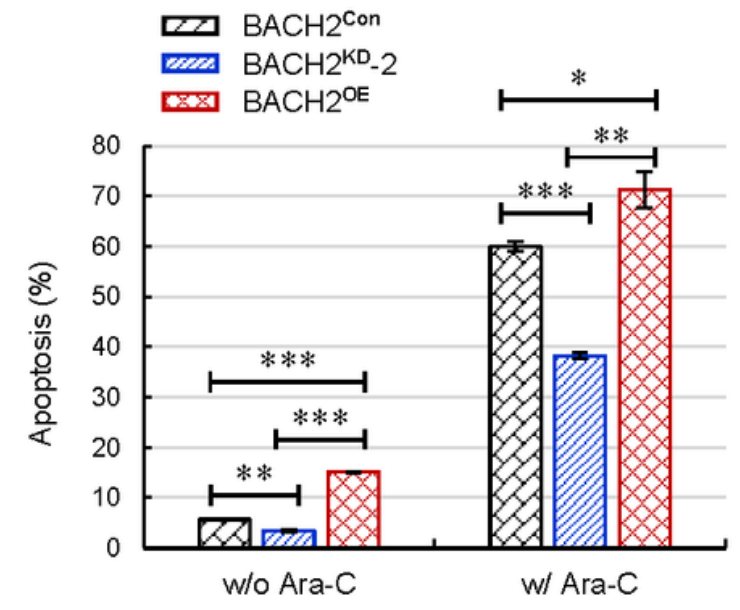




\section{Figure 4}

Decreased BACH2 expression confers chemo-resistant properties to p-ALL. (A) Children with ALL from prednisolone-resistant group $(n=27)$ contained lower $B A C H 2$ levels than those from prednisolonesensitive group $(n=66)$. (B) Children with B-ALL in prednisolone-resistant group $(n=20)$ contained lower $B A C H 2$ levels than those in prednisolone-sensitive group $(n=55)$. (C) Leukemia cell lines with lower $\mathrm{BACH} 2$ expression $(n=11)$ were more resistant to Ara-C compared to those with higher BACH2 levels $(n=9)$. The above results are shown as the mean \pm SEM. (D) Manipulated ALL cells were treated with or without Ara-C ( $20 \mathrm{nM})$, and cell survival was detected by staining cells with Annexin V/7-AAD at 48 hours. Representative FCM analyses are shown (left). The \% population of apoptotic cells in each group is shown as the mean \pm SD from 2 independent experiments (right). ${ }^{*} p<0.05 ;{ }^{* \star} p<0.01 ;{ }^{* \star} p<0.001$ (vs control group; Student t test). w/, with; w/o, without. 
A
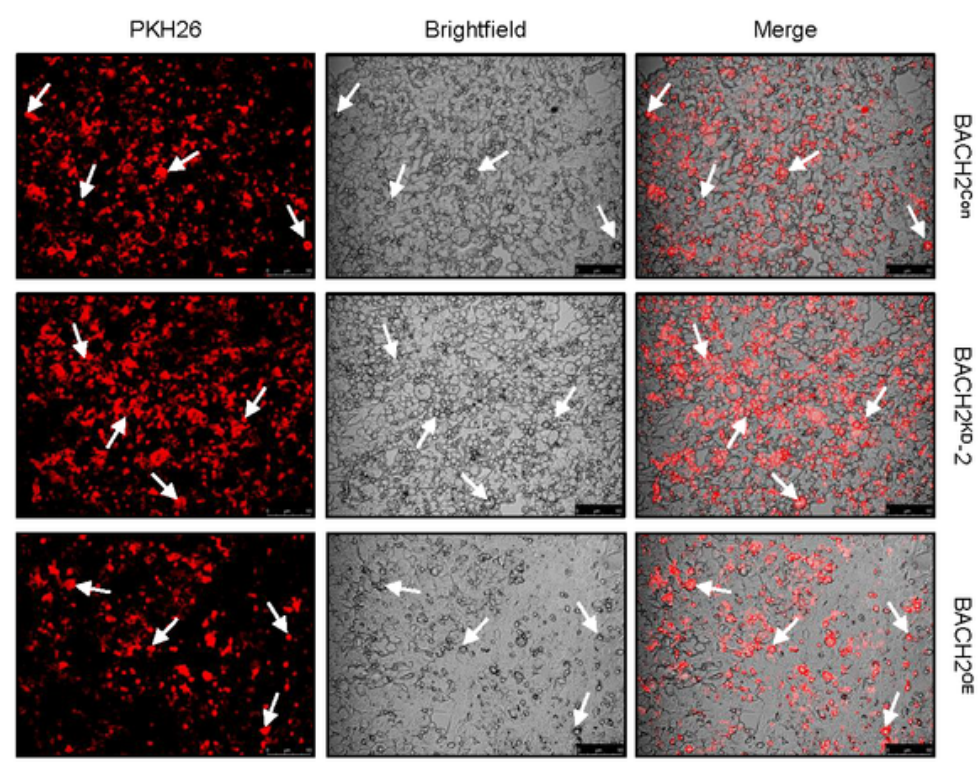

B

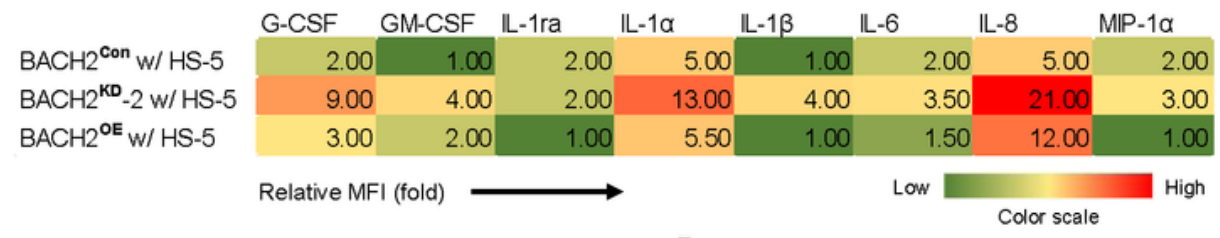

C

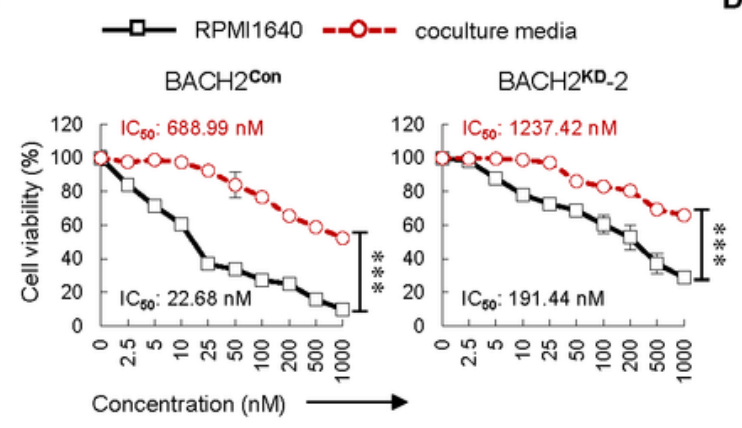

D
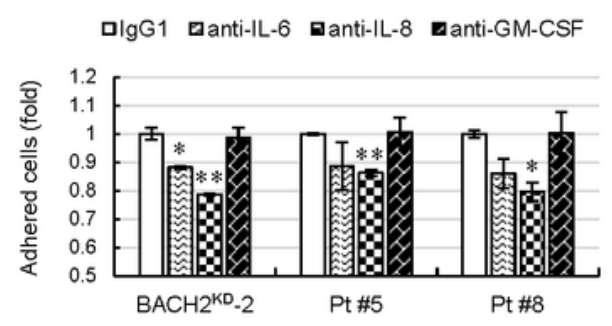

E

口Control पAra-C+IgG1 घAra-C+anti-IL-6 aAra-C+anti-IL-8 Ara-C+anti-GM-CSF

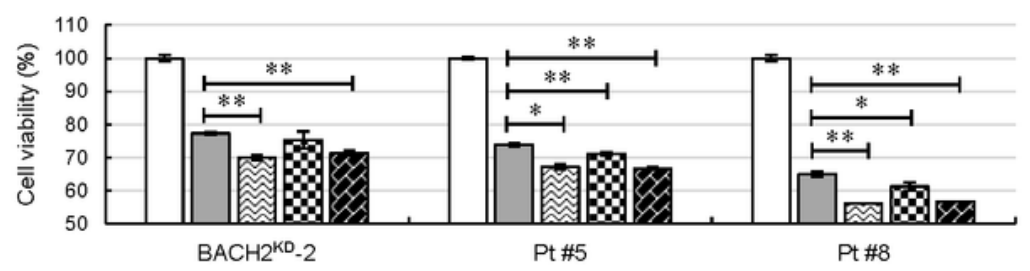

\section{Figure 5}

$\mathrm{BACH} 2$ silencing promotes cell adhesion and chemoresistance by altering stromal microenvironment. (A) Representative microscopic images of adherent leukemic cells in a coculture setting. Cells were stained with PKH26 prior to seeding onto a pre-established monolayer of HS-5 BMSCs. Scale bar, $50 \mu \mathrm{m}$. The arrows point to the representative leukemic cells adhered to BMSCs. (B) Relative mean fluorescent intensity (MFI) of multiple cytokines in coculture media under different coculture settings. The fold 
change in expression compared with the lowest value is indicated by the color intensity, with green representing reduced expression and red representing elevated expression. w/, with. (C) BACH2Con or BACH2KD-2 cells were cultured in complete RPMI1640 media or coculture media upon treatment of Ara-C for 48 hours. Cell viability was determined using MTT assays, which was normalized to nontreated control cells. IC50 values of Ara-C for $\mathrm{BACH} 2 \mathrm{Con}$ or $\mathrm{BACH} 2 \mathrm{KD}-2$ cells are indicated with red representing cells in coculture media and black representing cells in complete RPMI1640 media. (D) Primary BM cells from two p-ALL patients or BACH2KD-2 cells were stained with $\mathrm{PKH} 26$ prior to seeding onto a preestablished monolayer of HS-5 BMSCs. PKH26-stained cells were allowed to adhere for 4 hours with neutralizing antibodies of IL-6 $(0.5 \mu \mathrm{g} / \mathrm{ml})$, IL-8 or GM-CSF $(1 \mu \mathrm{g} / \mathrm{ml})$, or lgG1 antibody $(1 \mu \mathrm{g} / \mathrm{ml})$ as a negative control. PKH26 dye intensity of leukemic cells was normalized to those without neutralizing antibodies in each group. (E) Primary cells or BACH2KD-2 cells were treated with Ara-C (20 nM) for 48 hours with neutralizing antibodies of IL- $6(0.5 \mu \mathrm{g} / \mathrm{ml})$, IL-8 or GM-CSF $(1 \mu \mathrm{g} / \mathrm{ml})$, or IgG1 antibody (1 $\mu \mathrm{g} / \mathrm{ml}$ ) as a control. Cell viability was determined using MTT assays, which was normalized to nontreated control cells. Data are shown as the mean \pm SD from 3 independent experiments. ${ }^{*} p<0.05$; ${ }^{* \star} p<0.01 ;{ }^{* \star *} p<0.001$ (vs control group; Student $t$ test). 
A

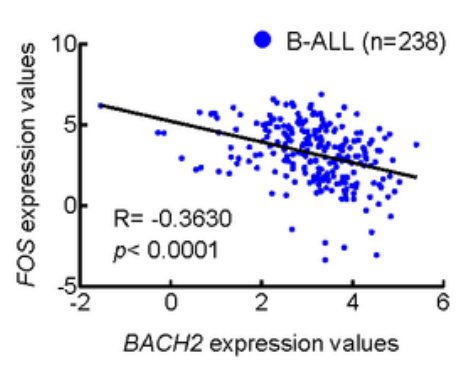

D
B

- $\mathrm{BACH} 2$

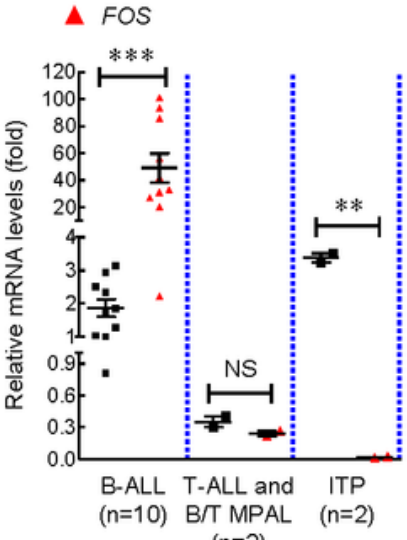

C

Pediatric ALL BM samples

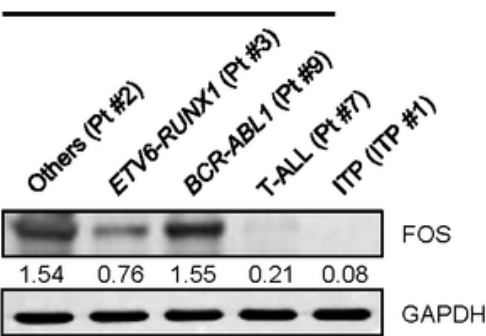

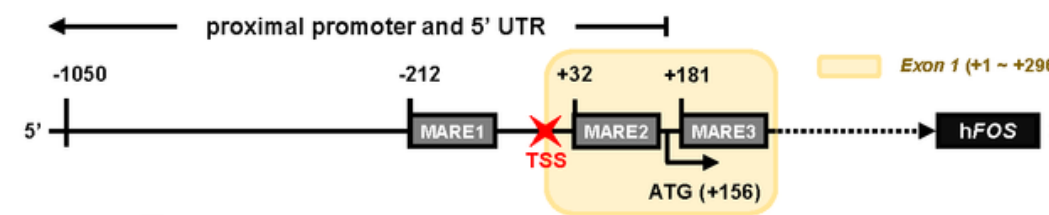

$\longrightarrow$ LUC $\rightarrow$ pGL3-basic

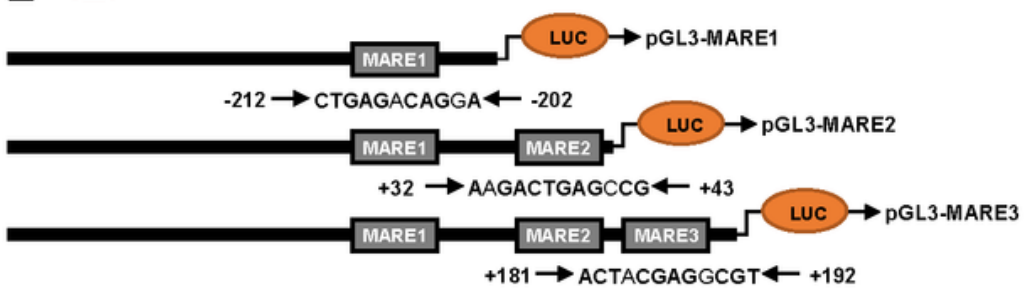

口pcDNA3.1 वpcDNA3.1-BACH2

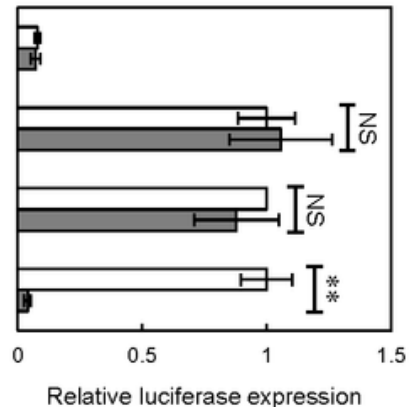

E

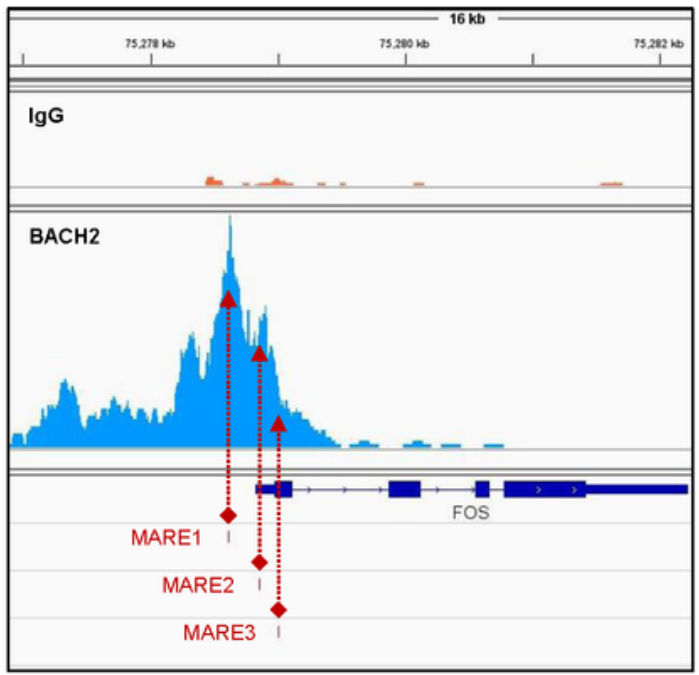

$\mathbf{F}$
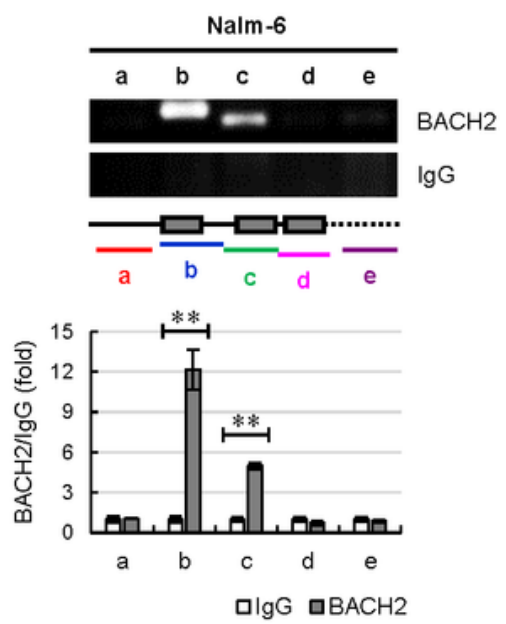

\section{Figure 6}

FOS is a downstream target repressed by $\mathrm{BACH} 2$ expression in pre- $\mathrm{B}$ leukemic cells. (A) Correlation analyses of $B A C H 2$ and FOS expression based on microarray data in newly diagnosed B-ALL $(n=238)$. $R$ value and $p$ value of are indicated. (B) BACH2 and FOS mRNA levels in $p$-ALL samples including B-ALL $(n=10), T-A L L$ and B/T MPAL $(n=2)$ with two ITP samples as negative controls. The fold change in expression compared with $\mathrm{BACH} 2$ levels of one $\mathrm{B}-\mathrm{ALL}$ patient is indicated. The results are shown as the 
mean \pm SEM. (C) Immunoblots of FOS in different subtypes of p-ALL with one ITP sample as a negative control. GAPDH was used as a loading control. (D) Three putative MARE binding sites within FOS proximal promoter (MARE1), 5' untranslated region (5' UTR, MARE2) and proximal Exon 1 (MARE3) along with truncated promoter constructs are indicated (left). 293T cells were transfected with truncated promoter plasmids with BACH2 expression plasmids or control plasmids (pcDNA3.1). An empty pGL3basic plasmid was used as a negative control. The Renilla luciferase reporter pRL-SV40 was used as an internal control for normalization. Luciferase activity was measured 48 hours after transfection. The results are presented as the relative luciferase activity compared with the cells with pcDNA3.1 expression. The results are shown as the mean \pm SD from 3 independent experiments. (E) Peak analysis by an IGV software following CUT\&Tag sequencing is indicated, with orange reads representing antibodies against control IgG and blue reads representing antibodies against BACH2. GRCh38 genome was used as a human reference genome. Peaks corresponding to MARE1, MARE2 and MARE3 are indicated (red arrows). (F) DNA fragments (a-e) amplified by PCR following CUT\&Tag assays are indicated (top), and the relative intensity of each band is shown from 2 independent validations (bottom). NS, not significant; ${ }^{*} \mathrm{p}<0.05 ;{ }^{* *} \mathrm{p}<0.01 ; * * * \mathrm{p}<0.001$. 
A Normal media

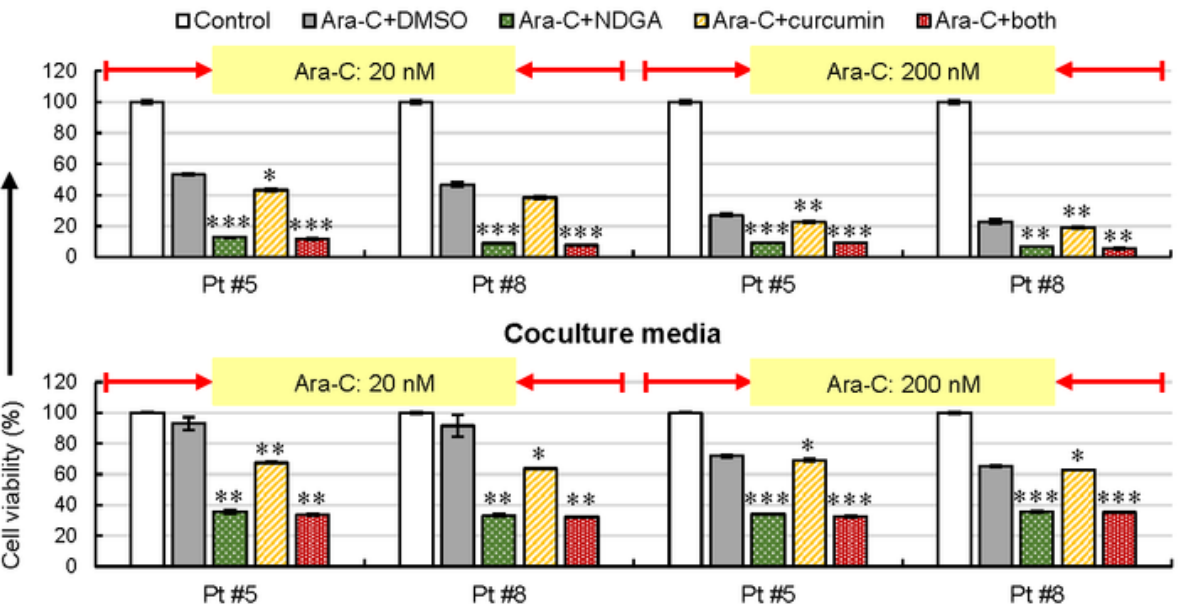

B

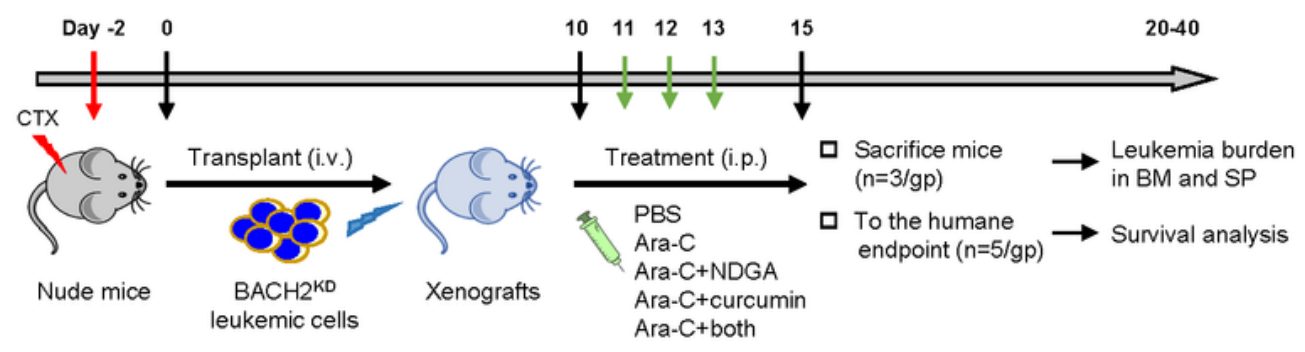

C

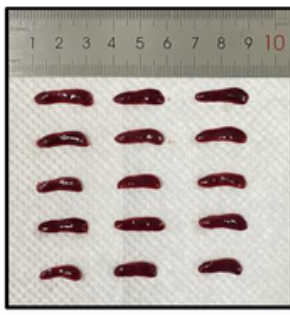

E

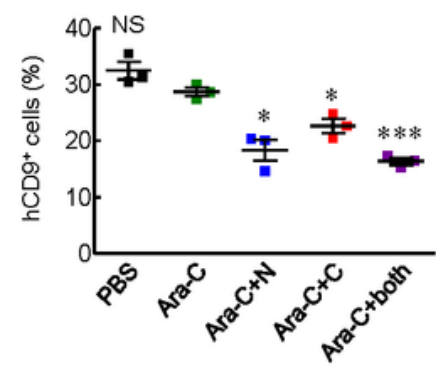

D

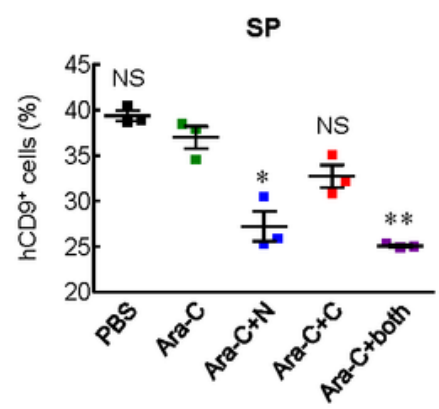

$\mathbf{F}$

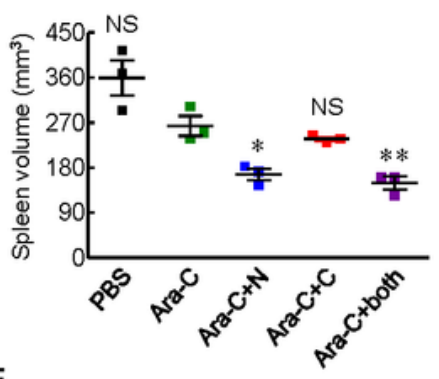

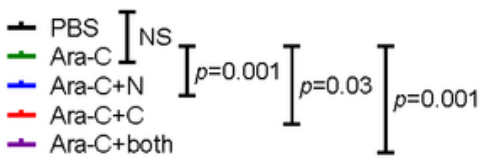

\section{Figure 7}

Chemical inhibition of FOS sensitizes leukemic cells to Ara-C in primary samples and xenografts. (A) Primary cells were cultured in complete RPMI1640 media (normal media, top) or coculture media (bottom) upon treatment of Ara-C at a low dosage of $20 \mathrm{nM}$ or a high dosage of $200 \mathrm{nM}$ for 48 hours in presence of NDGA, curcumin, or both. DMSO was used as a negative control. Cell viability was determined using MTT assays, which was normalized to nontreated control cells. The results are shown 
as the mean \pm SD from 3 independent experiments. ${ }^{*} p<0.05 ;{ }^{* \star} p<0.01 ;{ }^{* \star *} p<0.001$ (vs Ara-C+DMSO group; Student t test). (B) Experimental design for testing the efficacy of small molecule inhibitors of FOS (NDGA and curcumin) in vivo. (C) Spleens were isolated and photographed against a ruler (left). The size of spleens in each treatment group were measured (right). The results are shown as the mean \pm SEM. NS, not significant; ${ }^{*} p<0.05 ;{ }^{* \star} \mathrm{p}<0.01$ (vs Ara-C-treated group; Student $t$ test). N, NDGA; , curcumin. Human CD19+ (hCD19+) cells were isolated from SP (D) and BM (E) using anti-hCD19-MicroBeads. The percentage of hCD19+ cells in each organ was calculated, and the results are shown as the mean \pm SEM. NS, not significant; ${ }^{\star} p<0.05 ;{ }^{* \star} p<0.01$ (vs Ara-C-treated group; Student $t$ test). (F) Survival curve of xenograft mice treated with PBS (black), Ara-C (green), or a combination of Ara-C with NDGA (blue), or with curcumin (red), or with both (purple).

\section{Supplementary Files}

This is a list of supplementary files associated with this preprint. Click to download.

- Supplementaryfigures.pdf 